\title{
Spatial Representation and Cognitive Modulation of Response Variability in the Lateral Intraparietal Area Priority Map
}

\author{
Annegret L. Falkner, ${ }^{1,6}$ Michael E. Goldberg, ${ }^{1,2,3}$ and B. Suresh Krishna ${ }^{1,4,5}$ \\ ${ }^{1}$ Mahoney-Keck Center for Brain and Behavior Research, Department of Neuroscience, and 2Departments of Neurology, Psychiatry, and Ophthalmology, \\ Columbia University College of Physicians and Surgeons, New York, New York 10032, ${ }^{3}$ New York State Psychiatric Institute, New York, New York 10032 , \\ ${ }^{4}$ Bernstein Center for Computational Neuroscience, 37077 Goettingen, Germany, ${ }^{5}$ Cognitive Neuroscience Laboratory, German Primate Center, 37077 \\ Goettingen, Germany, and ${ }^{6}$ Department of Neuroscience, New York University School of Medicine, New York, New York 10016
}

The lateral intraparietal area (LIP) in the macaque contains a priority-based representation of the visual scene. We previously showed that the mean spike rate of LIP neurons is strongly influenced by spatially wide-ranging surround suppression in a manner that effectively sharpens the priority map. Reducing response variability can also improve the precision of LIP's priority map. We show that when a monkey plans a visually guided delayed saccade with an intervening distractor, variability (measured by the Fano factor) decreases both for neurons representing the saccade goal and for neurons representing the broad spatial surround. The reduction in Fano factor is maximal for neurons representing the saccade goal and steadily decreases for neurons representing more distant locations. LIP Fano factor changes are behaviorally significant: increasing expected reward leads to lower variability for the LIP representation of both the target and distractor locations, and trials with shorter latency saccades are associated with lower Fano factors in neurons representing the surround. Thus, the LIP Fano factor reflects both stimulus and behavioral engagement. Quantitative modeling shows that the interaction between mean spike count and target-receptive field (RF) distance in the surround during the predistractor epoch is multiplicative: the Fano factor increases more steeply with mean spike count further away from the RF. A negative-binomial model for LIP spike counts captures these findings quantitatively, suggests underlying mechanisms based on trial-by-trial variations in mean spike rate or burstfiring patterns, and potentially provides a principled framework to account simultaneously for the previously observed unsystematic relationships between spike rate and variability in different brain areas.

\section{Introduction}

The allocation of visual attention and the selection of saccade targets are based on spatial priority maps in a network of regions including the lateral intraparietal area (LIP), frontal eye field (FEF), and superior colliculus (Fecteau and Munoz, 2006; Bisley and Goldberg, 2010; Bisley, 2011). Consistent with priority map model predictions, we recently showed that most neurons in LIP

Received Nov. 13, 2012; revised Aug. 15, 2013; accepted Aug. 29, 2013.

Author contributions: A.L.F., M.E.G., and B.S.K. designed research; A.L.F. and B.S.K. performed research; B.S.K. contributed unpublished reagents/analytic tools; A.L.F. and B.S.K. analyzed data; A.L.F., M.E.G., and B.S.K. wrote the paper.

A.L.F. was supported by a National Science Foundation Graduate Research Fellowship and the Ruth L. Kirschstein National Research Service Award. M.E.G. was supported by National Eye Institute Grants R24EY015634, R01EY01497, R01EY017039, and P30EY019007; and by the Keck, Dana, and Zegar Foundations. B.S.K. was supported by German Ministry for Education and Science Grant BMBF 01GQ0433 to the Bernstein Center for Computational Neuroscience, and by a Gatsby Foundation award. We thank Drs. Mo Osman, Moshe Shalev, and Girma Asfaw for veterinary care; Yana Pavlova for veterinary assistance; John Caban for machining; Glen Duncan for electronics; and Latoya Palmer for facilitating everything. We also thank Drs. Mark Churchland, Samu Mäntyniemi, and Andreas Linden for helpful discussions.

The authors declare no competing financial interests.

Correspondence should be addressed to either of the following: Annegret L. Falkner, 522 1st Avenue, New York, NY 10016, E-mail: annegret.falkner@nyumc.org; or B. Suresh Krishna, AKN, Deutsch Primatenzentrum, 4 Kellnerweg, 37077 Goettingen, Germany, E-mail: skrishna@dpz.eu.

DOI:10.1523/JNEUROSCI.5269-12.2013

Copyright $\odot 2013$ the authors $\quad 0270-6474 / 13 / 3316117-14 \$ 15.00 / 0$ are strongly influenced by surround suppression over long distances (Falkner et al., 2010). Planning a saccade toward a location in space enhances its relative priority both by increasing the mean spike rate of LIP neurons representing that location and by suppressing the mean spike rate of LIP neurons representing other locations. Increasing motivation both further enhances the mean spike rate of neurons representing the saccade target and suppresses the responses of neurons representing surrounding locations; related results have been reported by Louie et al. (2011).

Reducing neuronal response variability can improve the precision of a neural code (Paradiso, 1988; Scobey and Gabor, 1989; Vogels, 1990; McAdams and Maunsell, 1999). The Fano factor, defined as the ratio of spike count variance to the spike count mean, is a representative measure of firing rate variability across trials (Tolhurst et al., 1981). Fano factor reduction is associated with stimulus onset in many cortical areas (Churchland et al., 2010), motor preparation in premotor areas (Churchland et al., 2006), changes in attentional allocation (Mitchell et al., 2007; Cohen and Maunsell, 2009), and saccadic latency in area V4 (Steinmetz and Moore, 2010), and reveals additional information to that obtained from the mean spike rate alone (Churchland et al., 2010; Hussar and Pasternak, 2010; Steinmetz and Moore, 2010). 
Little is known about the factors that influence the Fano factor in LIP, and, more generally, there is a lack of a framework for understanding the relationship between spike count and spike count variability in the brain. Similar to other cortical areas, stimulus onset in the receptive field (RF) leads to a reduction in neuronal Fano factor in LIP (Churchland et al., 2010), and across-trial variability increases with time during a decisionmaking task (Churchland et al., 2011); both findings relate to events occurring in relation to the neuron's RF. Characterizing the variability changes across the entire LIP map requires studying response variability in relation to stimulus events or behavioral significance in the surround, and this has not been done in LIP, and only very sparsely in other brain areas (Steinmetz and Moore, 2010; Chang et al., 2012). Furthermore, the influence of reward expectation on response variability has not been studied, to our knowledge, in any brain area. Here we demonstrate and quantitatively characterize the systematic variability changes across the LIP priority map during a visually guided delayed saccade task. We also demonstrate a global reduction in variability with increased expected reward. Our quantitative modeling of the LIP Fano factor and our use of a negative-binomial model to capture the underlying spike count distributional changes may be a useful framework to similarly characterize mean and Fano factor variations simultaneously in other brain areas.

\section{Materials and Methods}

Results in this study come from novel variability analyses performed on datasets whose mean spike rate properties we have previously described (Falkner et al., 2010). We therefore only briefly summarize the experimental procedures and methods here before describing the analysis procedures that are novel to this article. More details are available in the original publication (Falkner et al., 2010). We used three male rhesus monkeys (Macaca mulatta, $8-12 \mathrm{~kg}$ ). All experimental protocols were approved by the Animal Care and Use Committees at Columbia University and the New York State Psychiatric Institute, and complied with the guidelines established by the Public Health Service Guide for the Care and Use of Laboratory Animals. After locating the intraparietal sulcus using an MRI scan, we used standard surgical techniques to implant a headholding device, a recording chamber over the intraparietal sulcus allowing access to LIP, and a subconjunctival search coil to record eye movements. We used three recording cylinders: monkey D, left hemisphere; monkey I, right hemisphere; and monkey Z, right hemisphere. The other hemispheres in monkeys I and $\mathrm{Z}$ had been used in other LIP recording experiments. We used the REX/MEX/VEX system developed at the National Eye Institute Laboratory for Sensorimotor Research for behavioral control, visual stimulus display, and data collection using a Dell Optiplex PC running QNX (REX and MEX) and Windows 2000 (VEX). During recordings, the monkeys sat in a dimly illuminated room with their head fixed. Visual stimuli were back-projected onto a screen that stood $75 \mathrm{~cm}$ away using an LCD projector $(75 \mathrm{~Hz}$; CP-X275, Hitachi). We used a photodiode to register the actual times for stimulus onsets and offsets. Fixation point and saccade target stimuli were $0.3^{\circ}$ wide colored squares, and distractors were $1.5^{\circ}$ wide white squares. In each session, we introduced a glass-insulated tungsten electrode (Alpha Omega Engineering) electrodes through a guide tube positioned in a 1 $\mathrm{mm}$ grid (Crist Instruments) and recorded well isolated single units from LIP. Units were isolated while the monkeys performed a passive fixation task as white spots flashed sequentially at different locations in the visual field. We amplified, filtered, and discriminated action potentials using an amplitude window discriminator (MEX software). We considered neurons to be in LIP if they showed a consistent visual, delay period, and/or saccade-related response during the memory-guided saccade task or were located between such neurons in that electrode penetration. Every neuron responded to the abrupt onset of a visual stimulus in its RF.
Tasks

We studied the responses of 105 LIP neurons in three monkeys (43 in monkey Z, 26 in monkey D, 36 in monkey I). Our dataset consists of LIP neurons with systematically mapped RFs based on their clear visual response to a briefly flashed spot. For each neuron, we first identified the RF center during a passive fixation task using a series of briefly flashed stimuli to identify the location that elicited the maximal response. For most neurons, the monkeys then performed a memory-guided saccade task to the neuron's RF to further characterize the neuron's baseline properties in this standard task. The monkeys then performed several variants of the delayed visually guided saccade task, as described previously. We describe results from three tasks in this study. The variable number of cells in each task does not represent a subselection of the data, but reflects the fact that is some cases we were unable to maintain neuronal isolation throughout the period needed to obtain data using all three tasks. The target-mapping task (Figs. 1, 2, 3, 4) began with the appearance of a central red fixation spot; $500 \mathrm{~ms}$ after the monkey fixated the central spot, a saccade target appeared at a location randomly chosen from 80 possibilities (on a $40^{\circ} \times 40^{\circ}$ grid with $5^{\circ}$ spacing). A distractor flashed briefly for $<50 \mathrm{~ms}$ (two or three video frames in $\sim 90 \%$ of trials) at the center of the RF $500 \mathrm{~ms}$ after the saccade target appeared. The fixation spot disappeared $550 \mathrm{~ms}$ after distractor onset; this was the cue for the monkey to make the saccade (go-cue) within $400 \mathrm{~ms}$. Monkeys had to keep their eyes within a $3^{\circ} \times 3^{\circ}$ window until the cue to make the saccade appeared, and within a $4.5^{\circ} \times 4.5^{\circ}$ window around the saccade goal afterward. If the monkey's eye was in the window from 400 to $500 \mathrm{~ms}$ after the go-cue, the monkey received a drop of water or juice as reward. In control fixation trials performed both in a separate block and also interleaved (but occurring at low probability) with the saccade trials, the saccade target appeared right on top of the fixation point (without any noticeable flicker, since saccade targets and the fixation point had the same size) and the monkey simply maintained fixation at the fixation point to earn the reward.

In the cued reward task (Figs. 5, 6), we cued the monkey to expect either a large or a small reward randomly on each trial by changing the saccade target color (with one color each day representing large reward, and another color representing small reward). For most neurons, we ran a control task before this where the saccade target could be one of two different colors, but each was associated with the same reward to confirm the lack of color selectivity in the neuron. We conducted the task in two blocks: in the first block, the distractor appeared at the RF center, and the saccade was made to the suppressive surround; and in the second block, the saccade was made to the RF center, and the distractor appeared in the suppressive surround. Actual reward magnitudes were chosen daily based on the monkey's satiety level and his behavioral sensitivity to the difference in reward sizes, and were $\sim 1: 6$ on average. The task stimuli and timings in the cued reward task were otherwise identical to that in the target-mapping task, with the minor difference that the delay period lasted for $1000 \mathrm{~ms}$ rather than $1050 \mathrm{~ms}$. The final task we used was the memory-guided saccade task (Fig. 7) to the surround. This was identical to the target-mapping task except that the saccade target disappeared 50 $\mathrm{ms}$ after onset, and so the monkey had to make a memory-guided saccade rather than a visually guided saccade. Additionally, the delay period for this task again lasted for $1000 \mathrm{~ms}$.

\section{Data analysis}

All data analysis programs were written in MATLAB (MathWorks). We computed population-averaged spike rate peristimulus time histograms (PSTHs) by first obtaining PSTHs from each neuron and then finding the mean of these PSTHs. To quantify variability, we primarily use the Fano factor because it disambiguates the changes in variance of spike count from the associated changes in mean spike count. For example, for a Poisson counting process, the variance is always equal to the mean and therefore varies along with the mean. However, the Fano factor remains equal to 1 , revealing that variability does not change relative to Poisson expectations even as the mean changes. For the negative-binomial model, which is an extension of the Poisson counting process that we use where the variance is larger than the mean, the Fano factor and the mean spike count fully characterize the distribution of spike counts. However, 
we also discuss the results in terms of changes in the variance of spike counts to provide a clearer picture.

A procedure similar to that for the PSTHs of mean spike count was used to plot the peristimulus temporal evolution of the Fano factor: we refer to these plots as PSTFs. Unless otherwise specified, Fano factors were computed over $100 \mathrm{~ms}$ windows by dividing the variance of the spike counts within the $100 \mathrm{~ms}$ window by the mean spike count. A value of $100 \mathrm{~ms}$ is a plausible value for a counting window and, more importantly, keeps our analyses consistent with other recent articles on the Fano factor (Mitchell et al., 2007). Fano factors were calculated only when there were at least three trials and the mean spike count was $>0$. For the target-mapping task, we calculated the Fano factor (and spike count) independently for each target location. Population average PSTFs for Fano factors were calculated using partially overlapping $100 \mathrm{~ms}$ windows, sliding forward by $25 \mathrm{~ms}$ from one bin to the next. As a result, the Fano factor may appear to change in anticipation of the stimulus; however, this is an artifact of the large bin width used.

Validation of Fano factor analyses. Because the distributions in our data revealed significant deviations from normality (Jarque-Bera test) in the vast majority of cases, we use the median Fano factor rather than the mean as the summary statistic and accordingly use the Wilcoxon signedrank test rather than the $t$ test for all pairwise comparisons as well as the rank-based Friedman test rather than the repeated-measures ANOVA. However, all our main results remain qualitatively unchanged even when using the parametric alternatives. We used the Akaike information criterion (AIC) statistic to perform systematic comparisons between models for the data from the target-mapping task. We used all correct trials (where the monkey earned a juice reward) for our analyses. We also repeated our analyses after eliminating trials where the monkey made a microsaccade during the fixation period between saccade target onset and the go-cue: this did not change our results and conclusions meaningfully.

When averaging Fano factors from different locations (in the targetmapping task) and across different time windows (the multiple nonoverlapping $100 \mathrm{~ms}$ windows that compose the epoch of analysis), we calculated the Fano factor for each location and/or window and then averaged the Fano factors. The Fano factor is a ratio, and the direct estimation of a ratio is well known to be biased, meaning that the expected value of the ratio (or the "mean of ratios") calculated in finite samples does not equal the true value in the population (Rao, 2002). In simulations from the negative-binomial model (which characterizes our data well), we found that a "ratio-of-means" approach (where the variances and the means for each time window and/or location were averaged independently and then the average Fano factor was calculated as the ratio of this average variance to the average mean) was generally less biased and performed as well as other corrected estimators like the jackknife estimator and Tin's corrected estimator (Rao and Rao, 1971). However, this is a model-dependent conclusion, since, in general, this estimator can also be biased (Rao, 2002). For consistency with other recent analyses (Mitchell et al., 2007), we present our results using the standard mean-of-ratios estimator, but we verified that the patterns in our data remain robust when calculated using the ratio-of-means estimator. We also verified our conclusions using the entire time window of analysis (rather than as the average of the $100 \mathrm{~ms}$ component windows; Figs. 2, 4). The Fano factor for these longer windows is expected to be much larger than that of the Fano factor calculated as the average of the component $100 \mathrm{~ms}$ windows possibly because of autocorrelation and fractal fluctuations in the spike train, as has been shown for other brain areas (Teich et al., 1997; Oram et al., 2001; Averbeck and Lee, 2003; Orer et al., 2003; Osborne et al., 2004; Churchland et al., 2006). We emphasize that the window sizes we used for the analysis we presented here (100 ms) as well as for the validation $(100-500 \mathrm{~ms})$ ensured that it would be extremely unlikely that physical reasons like the discretization of spike times or a refractory period would produce a Bernoulli-distributed spike count distribution with 0 or 1 spike on every trial. Therefore, the limitation of the Fano factor to values $<1$ in the case of the Bernoulli distribution does not apply to our analyses.

Multiplicative model. We used the general linear model $F=a_{1} M+a_{2} D$ $+a_{3} M D+a_{4}$, where $a_{1}$ to $a_{4}$ are the coefficients; $M$ and $F$ are, respec- tively, the mean spike count and Fano factor within the predistractor epoch; and $D$ is the distance of the saccade target from the RF (Fig. 4). We used the mean spike count rather than the mean spike rate as the regressor for the analyses in Figure 4 because they lead to more interpretable parameters. This is because when spike counts are distributed according to a Poisson distribution, the distribution has a Fano factor of 1, independent of mean spike count, since the variance $(V)$ of spike counts is equal to the mean spike count $M$. However, this is not true for mean spike rate: if the duration of the counting window is $T$, then $V=V / T^{2}$, while the mean spike rate $=M / T$. Our data show Fano factors that are well above 1 (indicating a variance larger than the mean), and this can in principle be captured by different versions of what are called overdispersed Poisson models that allow for Fano factors well above 1. Once again, in these models, the Fano factor shows readily interpretable variations with mean spike count, but not with mean spike rate.

To test the efficacy of our general linear model, we compared it with other models where we allowed different coefficients for different target-RF distance zones. In all cases, the single multiplicative model performed better in the majority of neurons. For example, in 58 of 72 neurons, the single multiplicative model with four free parameters had a lower AIC statistic (indicating a "better" model) compared with the more detailed model where there were three different sets of four free parameters for each target-RF distance zone (target-RF distance between $10^{\circ}$ and $20^{\circ}$, between $20^{\circ}$ and $25^{\circ}$, and $>25^{\circ}$ ). Similarly, we also tested models where rather than having the Fano factor increase linearly with mean spike count for a fixed target-RF distance, the Fano factor either remained constant or included a dependence on the square of the mean spike count. Again, the linear model performed best in most neurons.

To test how well the linear model fit the observed data after accounting for the expected stochastic variability in neuronal spiking, we used a model-based approach. Assuming that a negative-binomial model held true, we estimated the parameters of the best-fitting negative-binomial model for each target location using the following relationships: scale parameter, $\theta=a_{2} D+a_{4}+\left(a_{1}+a_{3} D\right) M-1=$ $F_{\text {model }}-1$; and shape parameter, $k=M / \theta$ (Lindén and Mäntyniemi, 2011), where $F_{\text {model }}$ is the Fano factor predicted from the model fit. We then simulated the same number of trials as in the data for each target location using the parameters estimated above and repeated this process 500 times to calculate a distribution for the $r^{2}$ value that would emerge if the data were really generated from the proposed model. The $p$ values for the observed $r^{2}$ value in the real data could then be estimated based on this distribution.

Negative-binomial model. For the analysis of the cued reward task data (Fig. 6), we used the method of moments to fit a negativebinomial distribution. The first two moments fully determine the best-fitting distribution, and this method performs as well as or better than the maximum-likelihood procedure when dealing with small samples. The distribution is characterized by a shape parameter $k$ and a scale parameter $\theta$ : the method of moments equates $\theta$ to $F-1$ and $k$ to $M / \theta$. We only did the fitting when we had at least 10 trials for both the small-reward and large-reward conditions, and additionally, the mean was not greater than the variance (i.e., the Fano factor was not $<1$, which was usually the case). When the mean is greater than the variance, the distribution is underdispersed compared with a Poisson distribution, and the negative-binomial distribution is no longer a useful model. We chose the negative-binomial model after testing the Poisson-Inverse-Gaussian model as an alternative to the negativebinomial model: the negative-binomial model provided a better account of both the target-mapping and cued reward data. We also examined the target-mapping and cued reward data for a larger than expected probability of zero counts (zero inflation) and did not find any evidence for it.

Burstiness and regularity. We used the burstiness/refractoriness index (BRI; Compte et al., 2003; Anderson et al., 2011) to characterize the burstiness of the spike train. Briefly, we estimated the average autocorrelation function (ACF) for all trials (in a given neuron and condition) and then subtracted the shuffle predictor, calculated as the cross-correlation function of each trial with trials other than itself. This removes correla- 
tions resulting from time-locked variations in firing rate that are retained across trials. The subtracted value was then divided by the SD of the shuffle predictor (for each value of lag) to produce a corrected ACF. The BRI was then defined as the average height of the corrected ACF over lags from 1 to $4 \mathrm{~ms}$. To characterize the regularity of the interspike intervals, we used the measures CV2 (Holt et al., 1996) and CV (Softky and Koch, 1993; Maimon and Assad, 2009) from the literature. Both measures attempt to correct for the effect of withintrial and across-trial variations in firing rate on the interspike interval distribution. CV2 estimates the normalized local difference between two successive intervals with the assumption that the firing rate does not change much over that time scale. CV estimates the local firing rate with the assumption that the temporal evolution of firing rate is identical across all trials, with the time-varying firing rate on each trial differing only by a scaling factor. Interspike intervals from periods with common firing rates are then collected together and analyzed separately. The details of the calculations can be found in the original publications.

\section{Results}

Fano factor systematically decreases from the surround toward the saccade goal

We previously showed that LIP neuronal spike rates were strongly reduced when monkeys planned delayed saccades to different locations outside the neurons' RFs extending up to $35^{\circ}$ from the RF center, exhibiting broad surround-suppressive effects (Falkner et al., 2010). Response variability is another important component of spatial representations in neural maps: in many circumstances, a reduction of response variability leads to a more precise spatial representation (Paradiso, 1988). We therefore first examined whether there is a systematic spatial variation of the Fano factor in LIP neurons with respect to the spatial distance between a delayed saccade target and the neuron's RF center (target-mapping task; Fig. $1 B$ ). Monkeys were required to maintain fixation on a central red cue for 500 $\mathrm{ms}$, after which a saccade target would appear at a location randomly chosen from a $40^{\circ} \times 40^{\circ}$ square grid (Fig. $1 B$, bottom). We henceforth refer to the distance of this saccade target location from the RF as the target-RF distance. After a delay of $1050 \mathrm{~ms}$, the fixation point disappeared and the monkey had to make a saccade to the target. During most trials, $500 \mathrm{~ms}$ following the onset of the saccade target, a distractor was briefly flashed at the RF center.

We first focus on the predistractor epoch in a $500 \mathrm{~ms}$ interval that precedes the onset of distractor-related activity ( $470 \mathrm{~ms}$ before to 30 ms after distractor onset) and analyze it using the average of five consecutive non-overlapping $100 \mathrm{~ms}$ bins. This time window allows us to explore the spatial effects of the target and is uncontaminated by the visual response to the distractor at the RF center. The Fano Falkner et al. (2010).
A

Visually guided saccade: variable target location

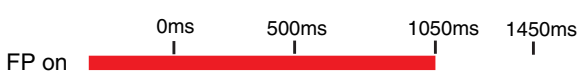

Target on

Distractor on

Saccade to target

C

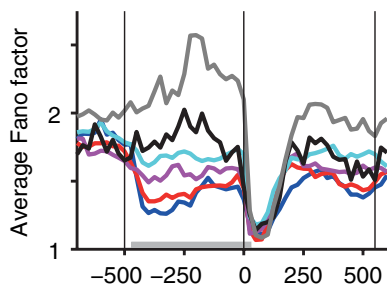

D
B

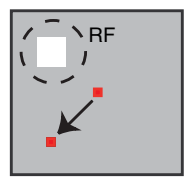

Saccade target locations

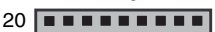

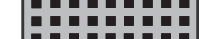

0 붐ำ -

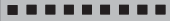

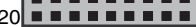

$\begin{array}{lll}-20 & 0 & 20\end{array}$

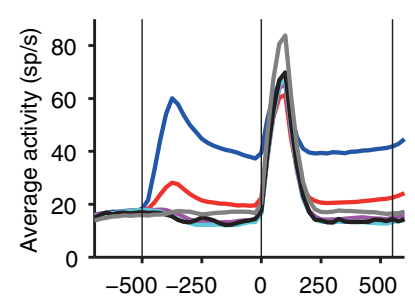

Time aligned to distractor (ms)
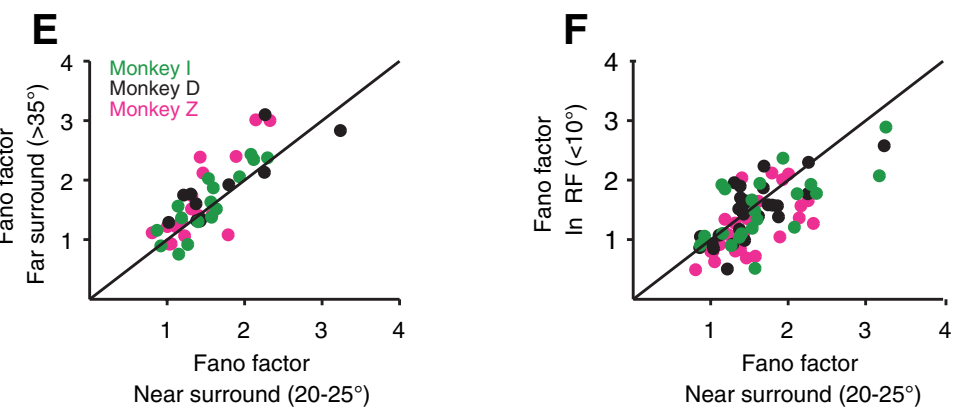

Figure 1. In LIP, the reduction in Fano factor becomes smaller with greater saccade target-RF distance. $\boldsymbol{A}, \boldsymbol{B}$, Timing $(\boldsymbol{A})$ and design $(\boldsymbol{B})$ of target-mapping task. Monkeys made delayed visually guided saccades to saccade targets randomly chosen from a $40^{\circ}$ $\times 40^{\circ}$ square grid of locations around the central fixation point $(\boldsymbol{B})$. A distractor was flashed briefly at the RF center $500 \mathrm{~ms}$ into the fixation in control fixation trials. C, Population average PSTF of the Fano factor indicates a greater reduction of the Fano factor in the epoch between target and distractor appearance for smaller target-RF distances (colors indicated in boxed legend). D, Population $20^{\circ}$ is not accompanied by changes in mean spike rate. The PSTF in Cand the PSTH in D were calculated in 100 ms windows stepping 作 $25 \mathrm{~ms}$. Vertical lines in $\boldsymbol{C}$ and $\boldsymbol{D}$ indicate the time of target, distractor, and go-cue onset. $\boldsymbol{E}, \boldsymbol{F}$, Pairwise comparisons confirm 列 $100 \mathrm{~ms}$ bins from -470 to $30 \mathrm{~ms}$ relative to distractor onset, gray bar in $C$ ) comparing saccades to the near surround ck, and magenta circles indicate neurons from monkeys $I, D$, and $Z$, respectively. Part of $\boldsymbol{B}$ was reproduced with permission from

factor decreases during the predistractor epoch for all target-RF distances compared with two separate control conditions. First, the Fano factor for all five target-RF distance zones (plotted using 100 ms bins in Fig. 1C) is smaller than the Fano factor in a $300 \mathrm{~ms}$ window before target onset $(p<0.0001$ for all five comparisons, signed-rank test, including for the farthest target locations where the population PSTF in Fig. $1 C$ suggests an increase). Second, the Fano factor for all five target-RF distance zones is also significantly smaller than the Fano factor during the predistractor epoch for the control fixation task (Fig. 1C, gray; $p<0.0005$ for all five comparisons, signed-rank test). The Fano factor during the control fixation task in the predistractor epoch is not significantly different from the Fano factor in the pretarget epoch (300 ms before target onset: $p=0.9508$, signed-rank test; $n=72$ neurons). 


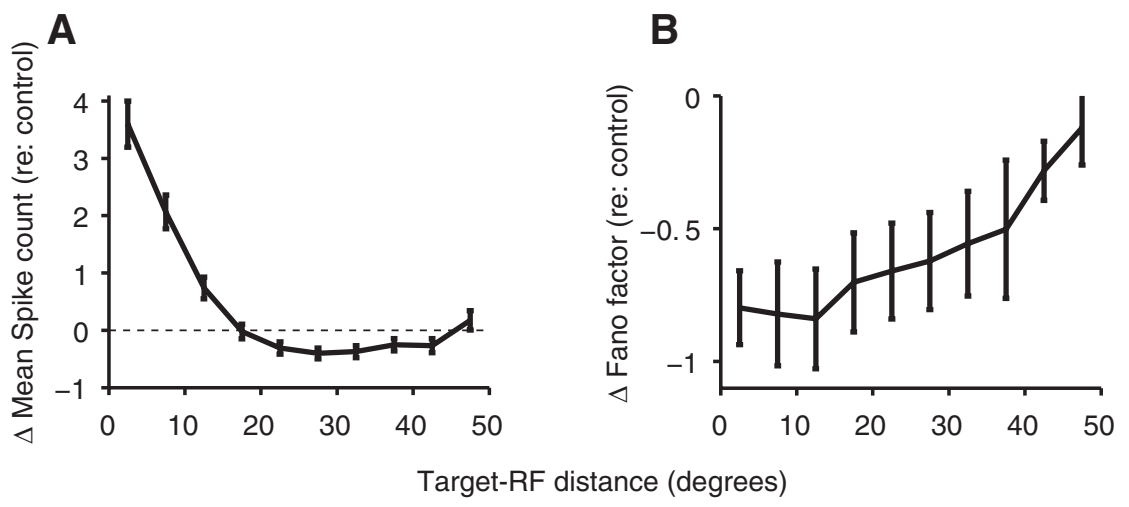

C

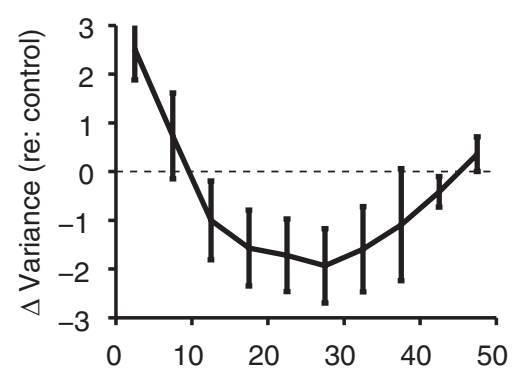

D

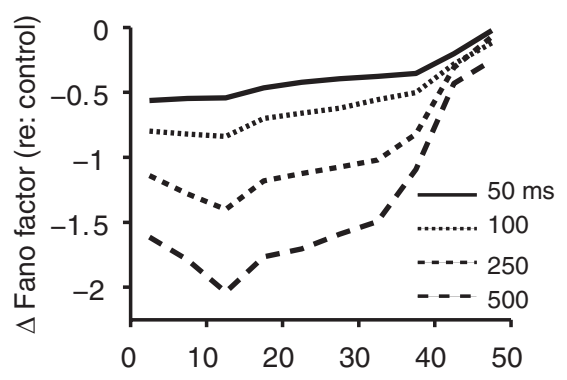

Target-RF distance (degrees)

Figure 2. Fano factors are reduced during the target-mapping task and show a steady decrease from the surround toward the saccade target. $\boldsymbol{A}-\boldsymbol{C}$, The values for the control fixation task were subtracted from that for the delayed saccade task with targets at different locations. $\boldsymbol{A}, \boldsymbol{C}$, In contrast, mean spike count shows a change from enhancement to suppression with increasing target-RF distance $(\boldsymbol{A})$, and a similar pattern is visible for the variance of spike counts $(\boldsymbol{C})$. D, The Fano factor increases with measurement window width but still shows the same pattern with target-RF distance: the plot for the $100 \mathrm{~ms}$ bin width is identical to that in $\boldsymbol{B}$. $\boldsymbol{A}-\boldsymbol{C}$, Error bars are SEMs. Fewer than five neurons had data for target-RF distances beyond $50^{\circ}$ (data not shown). Each point averages data from the bordering $5^{\circ} . \Delta$ represents change.

During the predistractor epoch, the Fano factor systematically decreases with decreasing distance of the saccade target from the RF (Fig. 1C). The maximal reduction of Fano factor occurs when the saccade target appears in the RF, and neurons whose RFs lie further from the saccade target show smaller reductions in Fano factors. For distances $>20^{\circ}$ from the RF center, these changes in Fano factor occur in the absence of a difference in the averaged spike rate during the same epoch (Fig. $1 D ; p>0.21$ for all three pairwise comparisons of distances $>20^{\circ}$, signed-rank test). To test these changes in Fano factor statistically across the population $(n=72$ neurons tested in the target-mapping task), we compared the trial-totrial variability of the response in the RF when targets appeared at near, intermediate, and far distances from the RF center. The Fano factor for targets $20-25^{\circ}$ from the RF center (the "near" surround) is significantly smaller than that for targets $>35^{\circ}$ from the RF center (the "far" surround; Fig. $1 E$; median decrease $=0.13, p=0.0047$, signed-rank test; $n=45$ neurons whose RF location allowed for distance sampling at $>35^{\circ}$ ), even though the spike counts in these epochs are not significantly different. Similarly, the Fano factor is significantly smaller for saccade target locations within $10^{\circ}$ of the RF center than it is for saccade targets in the near surround (Fig. $1 F$, median decrease $=0.20, p=0.0006$, signed-rank test $n=$ 72 neurons).

Thus, during the predistractor epoch of our visually guided saccade task, there is a systematically decreasing gradient of across-trial Fano factor in the LIP priority map from the surround toward the saccade target. This gradient is also clearly seen when we plot the average Fano factor as function of target-RF distance after subtracting the Fano factor for the control fixation task (Fig. $2 B ; p<0.0001$, Friedman's test for bins between $0^{\circ}$ and $35^{\circ}$; $n=61$ neurons with data for each bin). The corresponding plot for mean spike count (Fig. 2A) shows the pattern of enhancement for targets near the RF and a broad suppressive surround for targets further away that we previously reported (Falkner et al., 2010). The changes in Fano factor emerge from a relative increase of the variance compared with the mean spike count at increasing target-RF distances (Fig. 2C). This effect is not due to the choice of bin width over which the Fano factor was calculated: the observed pattern of Fano factor variation across target-RF distance holds true for a range of measurement windows from 50 to $500 \mathrm{~ms}$ during the predistractor epoch over which the Fano factor was calculated (Fig. $2 D ; p<0.0001$ for 50 and $100 \mathrm{~ms}$ windows; $p=0.0004$ for the 250 $\mathrm{ms}$ window; and $p=0.0119$ for the $500 \mathrm{~ms}$ window, Friedman's test; $n=61$ neurons with data for each bin).

We also observe a smaller reduction in Fano factor for larger target-RF distances in the postdistractor epoch in the average population PSTFs (Fig. 1C), even though the effect seems to be weaker and perhaps restricted to target-RF distances up to $35^{\circ}$. This was confirmed by pairwise analysis of the Fano factors in the period $300-500 \mathrm{~ms}$ following distractor onset. The Fano factor in the postdistractor epoch for saccade target locations within $10^{\circ}$ of the RF center is significantly smaller than that for saccade targets in the intermediate surround $\left(20-25^{\circ}\right.$ from the RF center; median decrease $=0.19 ; p=0.0021$, signed-rank test; $n=72$ neurons). Additionally, consistent with prior reports (Churchland et al., 2010), distractor onset at the RF center leads to a marked reduction in Fano factor to a value close to 1 (Fig. 1C), which is the expected value for a Poisson process with no additional across-trial variability. This reduction in Fano factor following distractor onset is transient and is considerably reduced by the time of the go-cue.

\section{Fano factor systematically increases with mean spike rate in the surround}

Studies reporting on the Fano factor face the challenge of disambiguating changes in Fano factor from the associated changes in mean spike rate. One approach that has been taken is to examine Fano factors after matching for mean spike rates across conditions (Churchland et al., 2010). We take a more direct approach here by explicitly modeling the relationship between the Fano factor and mean spike rate. For a Poisson distribution, which is the canonical model for neuronal spike counts, the Fano factor is independent of mean spike count. In our data, contrary to the 
expectation for a Poisson distribution, we find that the Fano factor in the predistractor epoch also systematically increases with mean spike rate. To examine the relationship between Fano factor and mean spike rate while minimizing the effect of target-RF distance, we first isolated locations with target-RF distances $>25^{\circ}$, where the mean spike rate ceases to show a systematic variation with distance in the population average (Fig. 1D). For each neuron, we divided saccade target locations $>25^{\circ}$ into three classes (low, intermediate, and high spike rate) based on their rankordered mean spike rate within the predistractor epoch. Locations with the lowest spike rates (Fig. $3 B$ ) are associated with the lowest Fano factor (Fig. 3A). The average Fano factor in the intermediate spike rate class is significantly larger than that in the low spike rate class (Fig. $3 C$; median increase $=0.16 ; p<0.0001$, signed-rank test; $n=72$ neurons), and the average Fano factor in the high spike rate class is significantly larger than that in the intermediate spike rate class (Fig. $3 D$; median increase $=0.25$; $p<0.0001$, signed-rank test; $n=72$ neurons). These results are not an artifact of using a $100 \mathrm{~ms}$ measurement window for the Fano factor because similar results were also obtained using a single $500 \mathrm{~ms}$ window for the entire predistractor epoch. The average Fano factor in the intermediate spike rate class with a $500 \mathrm{~ms}$ window is again significantly larger than that in the low spike rate class (median increase $=0.43 ; p=0.0006$, signed-rank test; $n=72$ neurons), and the average Fano factor in the high spike rate class is significantly larger than that in the intermediate spike rate class (median increase $=1.16 ; p<0.0001$, signed-rank test; $n=72$ neurons). The mean number of spikes in the $500 \mathrm{~ms}$ window were 4.37, 6.57, and 9.95 , respectively, for the low, medium, and high spike rate classes.

\section{Multiplicative interaction between target-RF distance and spike rate}

The increase in Fano factor with spike rate raises an apparent paradox about the relationship between mean spike rate and the Fano factor at different target-RF distances. Mean spike rate in the predistractor epoch increases as the saccade target is nearer to the RF, and this is associated with a greater reduction in Fano factor (an apparently inverse relationship). Yet our analysis above concluded that the Fano factor increases with mean spike rate within the predistractor epoch. To resolve this apparent paradox, we systematically examined the dependence of Fano factor on mean spike count at all target-RF distances. We divided the data into sequential $10^{\circ}$ target-RF distance windows centered $5-35^{\circ}$ from the RF center (Fig. $4 A, B$ ). For each neuron, within each target-RF distance window, we regressed the Fano factor upon the mean spike count, with each target location contributing one value for mean spike count and Fano factor. We chose to use mean spike count rather than mean spike rate as the regressor because they lead to more interpretable parameters (see Materials
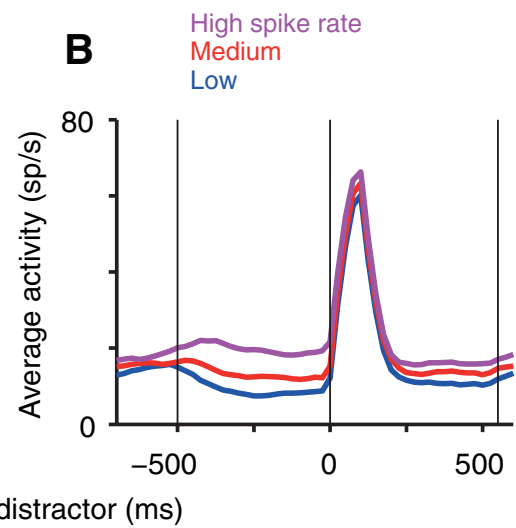

Time aligned to distractor (ms)

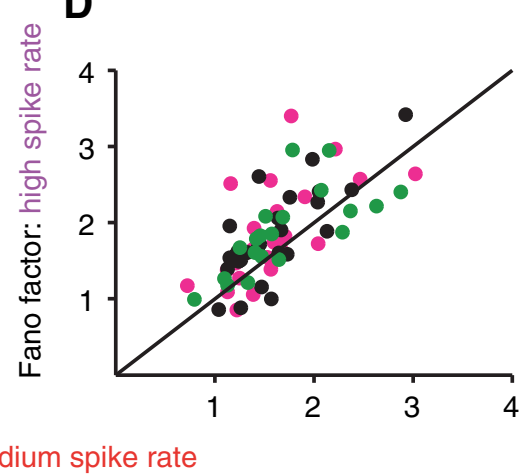

Fano factor: medium spike rate

Figure 3. LIP neuronal Fan o factor increases with mean spike rate in the surround. A, Population average PSTF of the Fano factor indicates a larger Fano factor in the epoch between target and distractor appearance for larger mean spike counts. Target locations ons). Two points in $\boldsymbol{D}$ ( $x$-axis: 1.63,2.39; $y$-axis: 4.98,4.09) were omitted from the display for better visibility. Figure format for as an Figure 1.

and Methods). We found a clear relationship between the mean slope and intercept parameters estimated by these regressions and target-RF distance. Both the mean slope and the mean intercept for a given target-RF distance window were obtained by averaging (across neurons) the fitted values obtained for each neuron for that particular target-RF distance window. The mean slope is significantly $>0$ for the bin centered at a target-RF distance of $10^{\circ}$ (mean slope $=0.12 ; p=0.005, t$ test for deviation from 0$)$, but not for bins centered at $5^{\circ}$ (mean slope $=0.07 ; p=0.1593$, $t$ test for deviation from 0 ). From here on, the mean slope relating Fano factor to mean spike count systematically increases with target-RF distance until $35^{\circ}$ (Fig. $4 A ; p<0.005$ for all target-RF distances, $t$ test for deviation from 0 ). This effect is accompanied by a reduction in the intercept with target-RF distance (Fig. $4 B$ ), as is also illustrated by the singleneuron example showing Fano factor versus mean spike count plots for two different target-RF distance windows (Fig. 4C). The mean intercept, because it describes the limit of the Fano factor as spike count approaches 0 , is a measure of the baseline Fano factor. The increase in slope (Fig. $4 A$ ) and the reduction in intercept (Fig. $4 B$ ) from $10^{\circ}$ to $35^{\circ}$ is statistically significant in the population ( $p<0.0001$, Friedman's test; $n=71$ neurons that had at least three locations in each target window). 
A

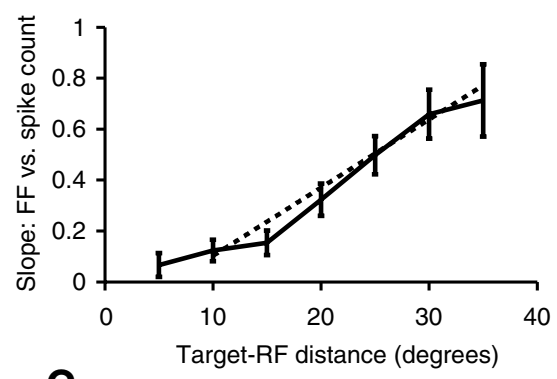

C

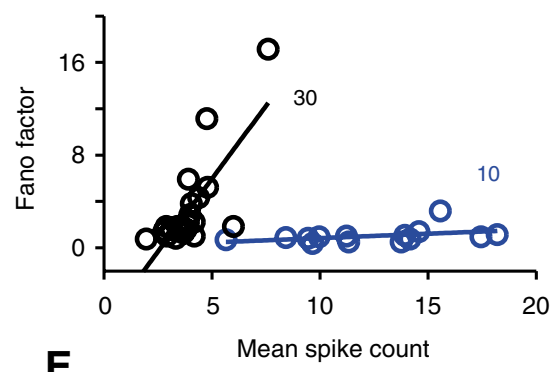

E

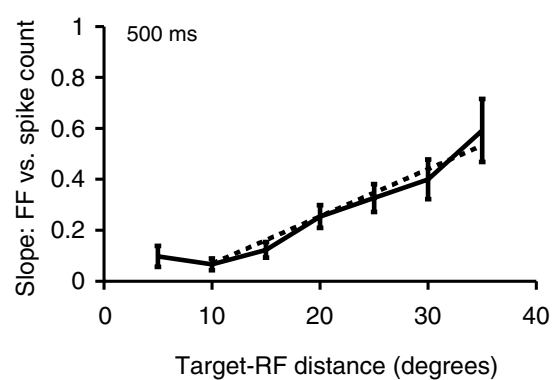

B

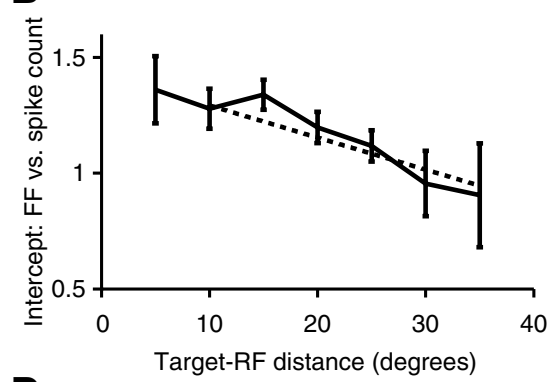

D
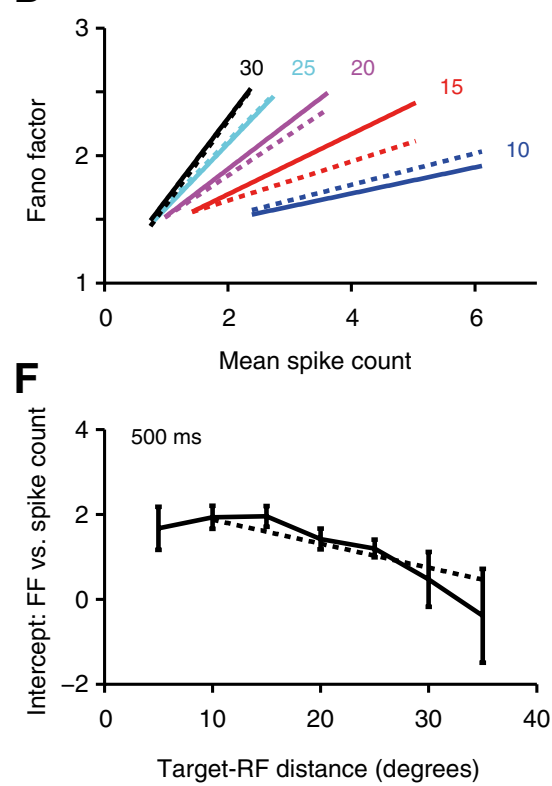

Figure 4. Target-RF distance interacts with spike rate in a multiplicative manner to affect the Fano factor (FF) in the surround. $\boldsymbol{A}, \boldsymbol{B}$, Regressing Fano factor on mean spike rate in different target-RF distance windows gives a slope $(\boldsymbol{A})$ that increases and an intercept $(\boldsymbol{B})$ that decreases with the distance of the target from the RF center (for targets at least $10^{\circ}$ away from the RF center). $\boldsymbol{A}, \boldsymbol{B}$, Slopes and intercepts as a function of target-RF distance calculated in partially overlapping $10^{\circ}$ target-RF distance windows, centered at $5^{\circ}$ intervals from $5^{\circ}$ to $30^{\circ}$ away from the RF center. The final window included only locations $>35^{\circ}$ away from the RF center. The dotted line is derived by averaging (across neurons) the predicted slope $(\boldsymbol{A})$ and intercept $(\boldsymbol{B})$ at each target-RF distance using a single linear multiple-regression model with a multiplicative interaction between spike rate and distance for all saccade target locations at least $10^{\circ}$ from the RF center. Error bars represent SEM. Variations of both slope and intercept with distance (solid lines) are statistically significant (Friedman's test, $p<0.0001)$. C, Single-neuron example of relationship of Fano factor versus mean spike count with superimposed regression line for target locations between $5^{\circ}$ and $15^{\circ}$ (blue) and between $25^{\circ}$ and $35^{\circ}$ (black) from the $R F$ center. $\boldsymbol{D}$, Relationship of Fano factor versus mean spike count for different target-RF distances from $10^{\circ}$ to $30^{\circ}$ (in steps of $5^{\circ}$ ), based on the average parameters of the general linear model in the population (solid lines) and the individual regressions for each target-RF distance window (dotted lines). The range of mean spike count values for each line comes from the mean $10-90 \%$ range of mean spike count values in the population for target-RF distance windows extending $5^{\circ}$ above and below the value for the line. $\boldsymbol{E}, \boldsymbol{F}$, Identical to $\boldsymbol{A}$ and $\boldsymbol{B}$ respectively, except that the Fano factor was calculated over the entire 500 ms predistractor epoch.

The increase in slope and the reduction in intercept with target-RF distance (Fig. $4 A, B$ ) appear to be linear starting from $10^{\circ}$ away from the RF center. We therefore predicted that a simple general linear model with a multiplicative interaction between spike count and distance could capture the observed Fano factor dependencies on target-RF distance and mean spike count, as follows: $F=a_{1} M+a_{2} D+a_{3} M D+a_{4}$, where $a_{1}$ to $a_{4}$ to are the coefficients. We performed such a regression for saccade target locations lying $10^{\circ}$ and beyond from the RF center, and predicted the variation in slope with distance in Figure $4 A$ as $a_{1}+a_{3} D$ and the variation in intercept with distance in Figure $4 B$ as $a_{2}+a_{4}$. Consistent with our prediction, the predicted variation agrees well with the actual variation measured in different distance win- dows (Fig. $4 A, B$, dotted lines). The results of this analysis thereby resolve the paradox by revealing the nature of the interaction between target-RF distance and mean spike count in determining the Fano factor: larger target-RF distances lead to larger slopes and smaller intercepts for the Fano factor versus spike count relationship. To further illustrate this, we calculated the average slope and intercept parameters across all neurons for each target-RF distance window based on both the general linear model (Fig. 4D, solid lines) as well as the individual regressions within each target-RF distance window (Fig. $4 D$, dotted lines), and then plotted the predicted relationship between Fano factor and mean spike count based on these averages.

Our results were also not dependent on our choice of a $100 \mathrm{~ms}$ measurement window: we show the similar results that were obtained when calculating the Fano factor over the entire $500 \mathrm{~ms}$ predistractor epoch (Fig. 4E, F; Friedman's test, $p \leq$ 0.0001 for both slope and intercept plots; $n=71$ neurons). The Fano factor calculated in this manner is expected to be larger than that of the Fano factor calculated as the average of the component windows due to autocorrelation and fractal fluctuations in the spike train, as has been shown for other brain areas (Teich et al., 1997; Averbeck and Lee, 2003). Consistent with that expectation, the Fano factor for the entire predistractor epoch is larger, but also highly correlated with the measure we used here based on nonoverlapping $100 \mathrm{~ms}$ windows (mean \pm SEM difference $=1.30 \pm 0.11$; linear regression for entire epoch vs $100 \mathrm{~ms}$ windows, both averaged across all target locations: mean \pm SEM slope $=2.2 \pm$ 0.21 ; mean \pm SEM intercept $=-0.6 \pm$ $0.34 ; r^{2}=0.62 ; n=72$ neurons). Further, all the results we presented above (the dependence of Fano factor on target-RF distance and mean spike count, as well as the multiplicative interaction between these two factors) remain true (with expected quantitative differences) when recomputed using the Fano factor and mean spike count for the entire predistractor epoch at once.

\section{Increasing expected reward has different effects on the Fano factor and mean spike rate}

We previously showed that increasing motivation by signaling a larger expected absolute reward on a subset of trials enhances the mean spike rate of neurons representing the saccade target and suppresses the distractor response of neurons whose RFs lie further away relative to trials in which a low reward is signaled (Falkner et al., 2010). Since neuronal variability can critically impact the effect of motivation on the LIP representation, we used the same dataset to examine the effects of increased motiva- 
A

Visually guided saccade with variable reward

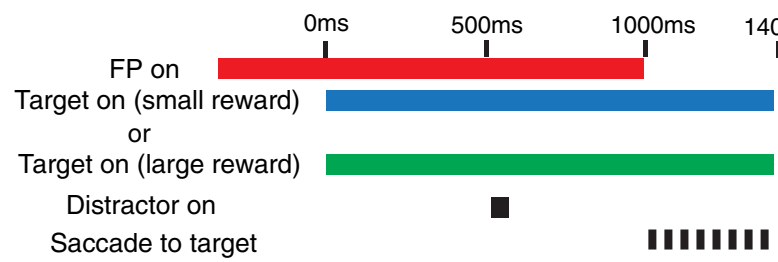

Target in RF

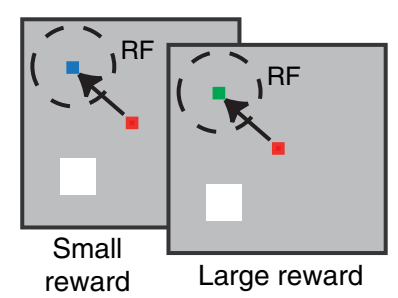

Distractor in RF

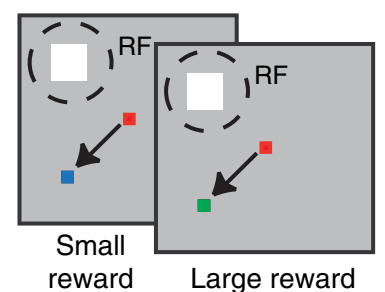

B Target in RF
C
Distractor in RF

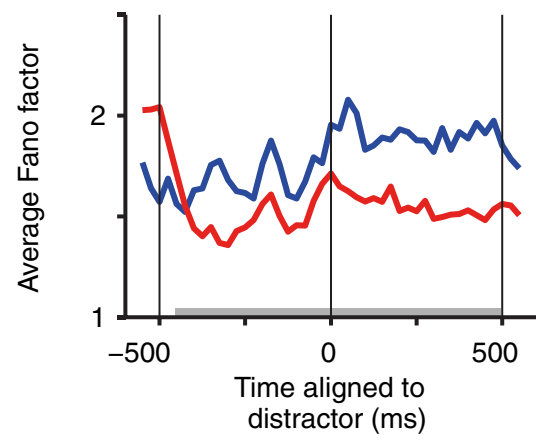

$\mathbf{F}$

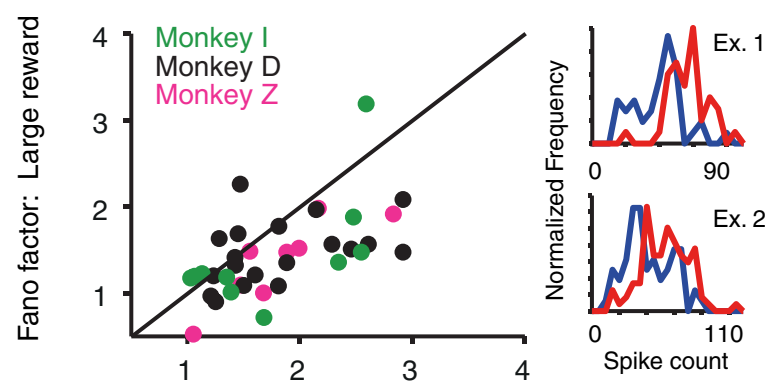

Fano factor: Small reward

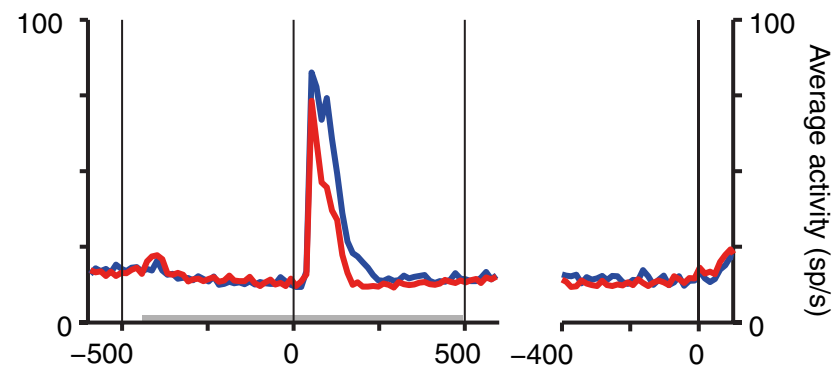

E
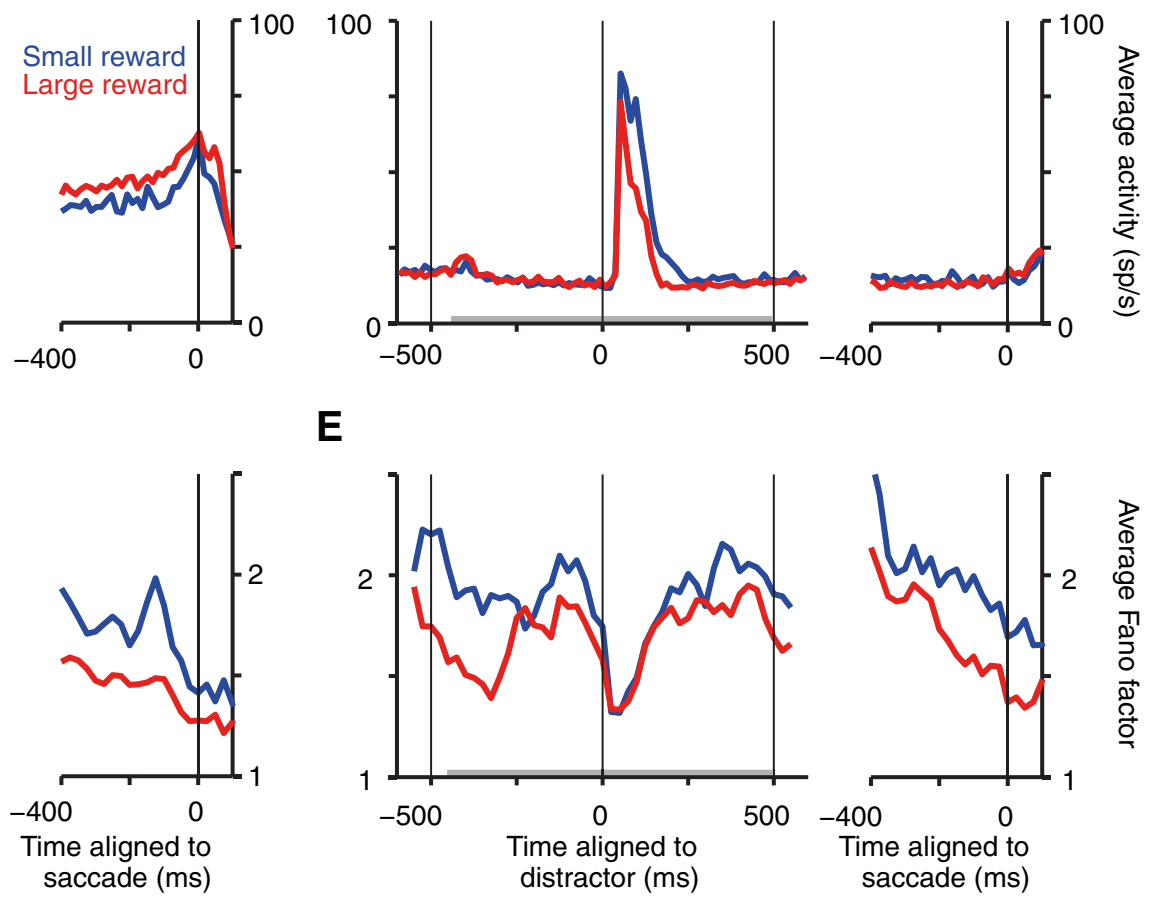

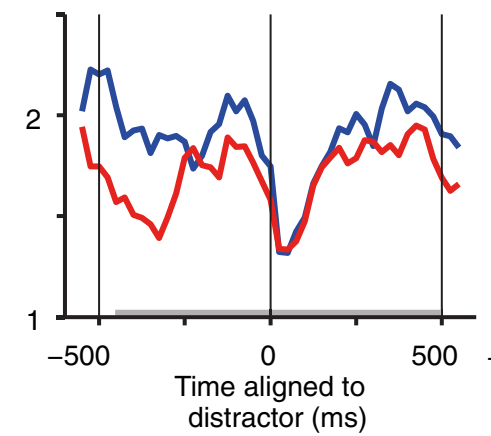

\section{G}

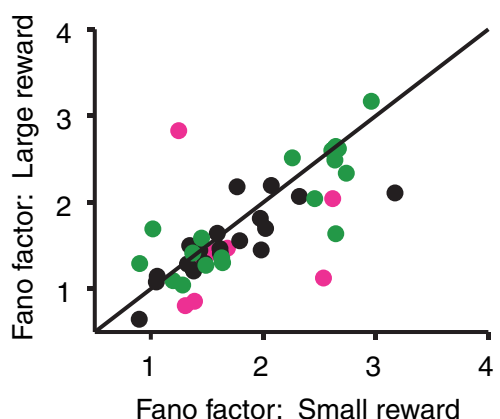

Fano factor: Small reward

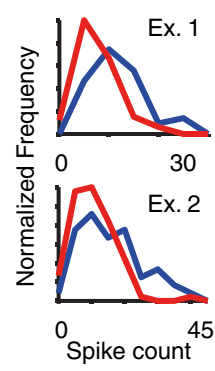

Figure 5. Increasing motivation via expected reward has a differing (push-pull) effect on mean spike rate for target and distractor responses but reduces Fano factor globally. $\boldsymbol{A}$, Timing (left) and schematic (right) of visually guided saccade task with variable reward. $\boldsymbol{B}-\boldsymbol{E}$, The PSTHs $(\boldsymbol{B}, \boldsymbol{C}$ and PSTFs $(\boldsymbol{D}, \boldsymbol{E})$ show data for large rewards in red and for small rewards in blue. Data in $\boldsymbol{B}, \boldsymbol{D}$, and $\boldsymbol{F}$ (left column) from target in RF, distractor in surround task condition, and data in $\boldsymbol{C}, \boldsymbol{E}$, and $\mathbf{G}$ from target in surround and distractor in RF task condition. $\boldsymbol{B}, \boldsymbol{C}$, Population average PSTHs of the spike rate (15 ms non-overlapping bins) show that increasing expected reward increases the mean target-response and decreases the mean distractor response. The left and right graphs in each panel show data aligned to distractor onset and saccade onset, respectively; the left graphs replicate a plot in Falkner et al. (2010) and are reproduced with permission. $D$, E, Corresponding PSTFs for the Fano factor, however, show a reduction in Fano factor when a larger reward is expected, independent of target location. Gray bar at bottom denotes time window for scatter-plot computation (F, G). PSTFs and PSTHs are as in Figure 1 C.F, $, \mathbf{G}, \mathbf{S c a t t e r}$ plot of data from each neuron (calculated as an average of non-overlapping $100 \mathrm{~ms}$ windows from $400 \mathrm{~ms}$ before to $500 \mathrm{~ms}$ after distractor onset, marked in gray in $\boldsymbol{B}-\boldsymbol{E}$ ) shows a significant decrease in Fano factor with increased motivation for both conditions ( $\boldsymbol{F}: p=0.0008, n=38 ; \boldsymbol{G}: p=0.0030, n=46$; signed-rank test). One point in $\boldsymbol{F}(x$-axis, 3.10; $y$-axis, 4.51$)$ and $\boldsymbol{G}(x$-axis, 4.91; $y$-axis, 3.36) was omitted from the display for better visibility. Right panels in $\boldsymbol{F}$ and $\boldsymbol{G}$ shows normalized frequency polygons of spike counts for two-example neurons with data from the small (blue) and large (red) reward conditions for each neuron, using the entire analysis window. With larger expected reward, for the neurons in the upper and lower plots, the Fano factor was reduced from 5.29 to 2.37 and 7.12 to 5.41 (target in RF) and from 2.78 to 1.99 and 5.42 to 4.26 (distractor in RF), respectively. 
tion on the Fano factor. The task (cued reward task; Fig. 5A) was a variant of the target-mapping task where either the saccade target appeared in the RF and the distractor at a single location in the suppressive surround (Fig. $5 B, D, F$ ) or vice versa (Fig. $5 C, E, G)$. These two conditions were tested in separate blocks. On randomly interleaved trials within each block, the saccade target color indicated either a large reward or a small reward on successful trial completion: monkeys learned the color associations rapidly during the first few trials of the session (Falkner et al., 2010). Increasing the expected reward enhances the response to the saccade target and decreases the response to the distractor in a "push-pull" manner (as reported earlier; Fig. 5B, C, population PSTHs). During the delay period ( $400 \mathrm{~ms}$ before to $500 \mathrm{~ms}$ after distractor onset), the spike rate increased, with a larger expected reward when the target was in the $\mathrm{RF}$ (median increase $=5.08$ spikes/s; $p<0.0001$, signed-rank test; $n=38$ ) and with a smaller expected reward when the distractor was in the RF (median increase $=2.46$ spikes $/ \mathrm{s} ; p=0.0011$, signed-rank test; $n=46$ ). In clear contrast, increased motivation decreased the Fano factor independent of whether the target or the distractor was in the neuron's RF, as indicated by the average population PSTFs (Fig. $5 D$, target; Fig. $5 E$, distractor in RF) and confirmed by the pairwise comparisons of Fano factors averaged during the delay period in the large- and small-reward conditions with a target in the RF (Fig. 5F; median decrease $=0.37, p=0.0008$, signed-rank test; $n=38$ ) and a distractor in the RF (Fig. $5 \mathrm{G}$; median decrease $=0.16, p=0.0030$, signed-rank test; $n=46)$. Singleneuron examples for each condition (Fig. $5 F, G$, right) further illustrate this by exhibiting the spike count distributions for small- and large-reward trials. Together, these data suggest that increasing motivation via reward has a global effect on variability, reducing Fano factor at widely separated locations on LIP's map. These results also further corroborate the increasing body of evidence indicating that changes in Fano factor can occur without concurrent variations in spike rate. Specifically, during the predistractor epoch when the distractor was in the RF, the mean spike rate did not change significantly with increased expected reward (median increase $=0.54$ spikes $/ \mathrm{s} ; p=0.8313$, signedrank test; $n=46$ ), but the Fano factor decreased with increased expected reward in the same epoch (median decrease $=0.19 ; p=0.0004$, signed-rank test; $n=46)$.

\section{A negative-binomial model fit captures the effects of expected reward on the spike count distribution}

Our description until now has focused on the mean and the Fano factor as summary statistics for the observed spike count distributions under different conditions. Characterizing the changes in the full spike count distribution, even using a purely phenomenological model, could prove very useful for several reasons. For example, it can justify the use of the mean and the Fano factor as summary statistics, allow the exploration of the statistical consequences of the observed changes for encoding and decoding, and enable productive comparisons between candidate generative models and neural data. The scatter plots (Fig. $5 F, G$ ) show that the Fano factors in our data are all generally well above 1 (proportion of neurons with Fano factor $\geq 1$ : with target in RF, small reward $=100 \%$, large reward $=89.5 \%$; with distractor in $\mathrm{RF}$, small reward $=95.7 \%$, large reward $=93.5 \%)$. The preponderance of values $\geq 1$ indicates that the spike counts are overdispersed compared with the expectation based on the standard Poisson distribution for spike counts. A standard way to capture such overdispersion is to use a negative-binomial model. This model is characterized by a shape parameter, $k$, and a scale pa- rameter, $\theta$, and these parameters can be estimated directly from the mean $(k \theta)$ and the Fano factor $(\theta+1)$, thereby fully characterizing the model. The negative-binomial model is a plausible phenomenological model for overdispersed spike counts because there are at least two candidate generative models that can produce it, based on trial-by-trial variations in the mean firing rate of the neuron (a doubly stochastic process; Churchland et al., 2011) or variations in burst occurrence probability and size (see Discussion). In the first scenario, if the negative-binomial measure proved to capture the data well, then the measure variance mean (equal to $k \theta^{2}$ ) would be equal to the variance of conditional expectation measure that has been used in recent publications (Churchland et al., 2011; Marcos et al., 2013): this measure characterizes the variability in the trial-by-trial input to a Poisson process by subtracting out the variability due to the Poisson process itself from the total variability. In our data, increasing expected reward led to reductions in this measure as well, just as it did for the Fano factor, both with the target in RF (median decrease $=9.74 ; p=0.0052$, signed-rank test; $n=38$ ) and with the distractor in RF (median decrease $=4.98 ; p=0.0064$, signedrank test; $n=46)$.

To examine whether the negative-binomial model could be used to characterize our data, we fitted a negative-binomial model with shape parameter $k$ and scale parameter $\theta$ to the data from the small- and large-reward conditions from each neuron where the variance was greater than or equal to the mean for both large and small expected rewards and there were at least 10 trials per condition. This restriction reduced the number of neurons to 35 (from 38) and 40 (from 46) for the target in RF and distractor in RF conditions, respectively. Since the model applies to the full distribution of spike counts across trials and therefore captures variations in the mean spike count and Fano factor simultaneously, we chose epochs where the differences in mean spike rate with expected reward did not vary substantially across time ( $400 \mathrm{~ms}$ before to $500 \mathrm{~ms}$ after distractor onset for the target in RF condition, and $470 \mathrm{~ms}$ before to $30 \mathrm{~ms}$ after distractor onset for the distractor condition) and found that the model captured the observed distributions; using a $\chi^{2}$ goodness-of-fit test, only 4 of 70 fits for the target in RF condition and 1 of 80 fits for the distractor in RF condition were significantly rejected at the 0.05 level (using the BenjaminiHochberg false discovery rate correction).

To visualize the adequacy of the fit, we used the following procedure: we counted the number of spikes on each trial within the epoch of interest to construct the distribution of spikes across trials (note that this is different from the averages across nonoverlapping $100 \mathrm{~ms}$ bins within the epoch of interest that we mostly used until now). We then made the assumption that the neuronal responses were independent from each other and across trials. With this assumption, even though we did not record simultaneously from every neuron in our dataset, we could analyze the data as if we had done so, and the mean of the responses from one randomly drawn trial each from every neuron could be used to approximate the mean spike count on a given trial across the population of recorded neurons. Repeating this mean calculation many times allowed us to estimate the full distribution of mean spike counts averaged across the population of recorded neurons. To test the model's ability to fit the data, we used the real recorded data to randomly sample the single-trial response from each neuron (Fig. 6, solid lines), and we simulated the single-trial response using each neuron's best-fitting negative-binomial distribution (Fig. 6, dotted lines). The close agreement between the solid and dotted lines in Figure 6 indicates that the model adequately cap- 
tures the true distribution of the recorded data. For one such comparison after 25,000 repetitions, a Kolmogorov-Smirnoff test between the distributions estimated using the real data and the model-based simulated data did not reveal a significant difference for either the large- or small-reward conditions with the target or the distractor in the RF (all $p>0.1$ ). However, the distributions for the high- and low-reward conditions were very significantly different from each other, with either the target or the distractor in the RF $(p<0.0001)$.

To further quantify the differences between the high and low reward condition distributions, we examined how the effects in the cued reward task translated to variations in $k$ and $\theta$ parameters of the fitted negative-binomial distributions. In the negative-binomial model, $M=k \theta$, $V=k \theta+k \theta^{2}$, and $F=1+\theta$. Thus, an increase in Fano factor should correspond to an increase in $\theta$, while an increase in the mean can come about through an increase in either $k$ or $\theta$ or in both. Consistent with this expectation, with the target in the RF, the scale parameter $\theta$, estimated for both small- and large-reward conditions in each neuron, decreased significantly with larger expected reward (median decrease $=1.38 ; p=0.0228$, signed-rank test; $n=35$ ), while the shape parameter $k$ increased significantly with larger expected reward (median increase $=5.06$; $p=0.0001$, signed-rank test; $n=35$ ). Similarly, with the distractor in the RF, $\theta$ again decreased significantly with larger expected reward (median decrease $=$ $0.56 ; p=0.0040$, signed-rank test; $n=40$ ), while $k$ increased significantly with larger expected reward (median increase $=$ 1.20; $p=0.0005$, signed-rank test; $n=40$ ). As a reference, the baseline median values in the small-reward condition were as follows: $\theta=4.06$ and $k=8.48$ with the target in RF; $\theta=1.71$ and $k=3.45$ with the distractor in RF. The larger parameter values with the target in RF reflect the larger width of the analysis window used in this condition.

Finally, using the same epoch used for the model fitting, we also analyzed whether reductions in Fano factor with increasing expected reward arise via a reduction in spike count variance or an increase in spike count mean. When the target is in the RF, the statistically significant decrease in Fano factor with increased expected reward (median decrease $=1.02 ; p=0.0324$, signed-rank test; $n=38$ ) results from a statistically significant increase in the mean spike count (median increase $=4.59 ; p<0.0001$, signedrank test; $n=38$ ) without any significant change in variance (median decrease $=7.58 ; p=0.4209$, signed-rank test; $n=38$ ); this is especially visible in the centered distribution in Figure $6 D$, where the distributions of the mean spike count for the smalland large-reward conditions appear to be superimposed after the mean is subtracted out. We emphasize though that the expectation based on a Poisson (or near-Poisson) count model is that the variance should increase in proportion to the mean, and therefore, one could equally well view the reduction in Fano via an
Target in RF

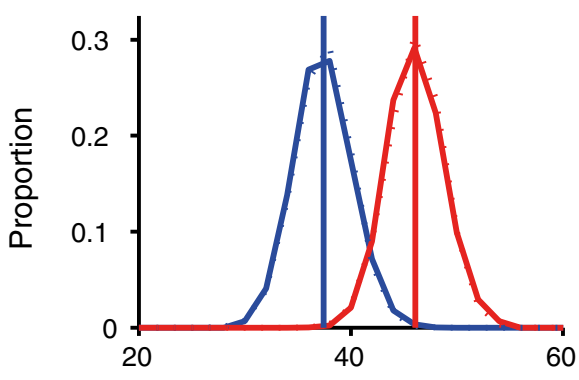

60

Centered

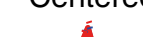

20
B

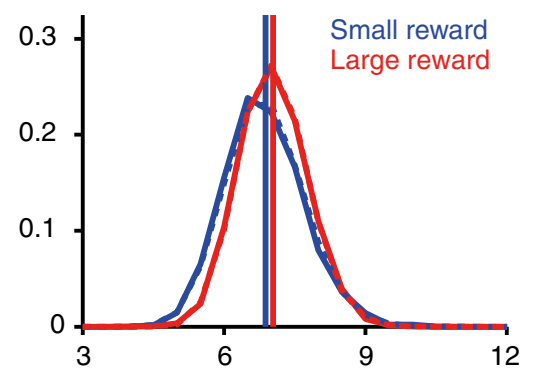

D

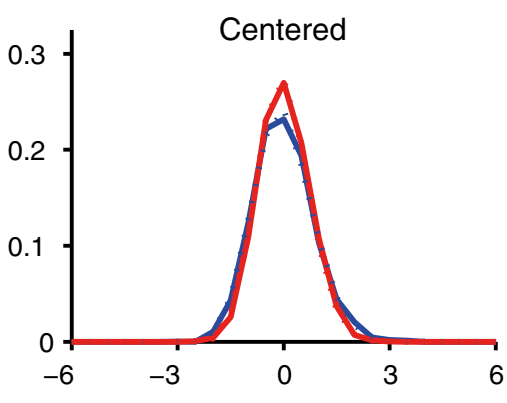

Mean spike count (all neurons)

Figure 6. A negative-binomial model fit captures the effects of expected reward on the spike count distribution. $A-D, B l u e$ and red indicate small and large rewards, respectively. Solid lines are frequency polygons of the distribution of mean spike count across 作 mly chosen trial from each neuron was used to compute a mean spike count, and the distribution of the mean spike count was stimated by repeating the process 25,000 times. Dotted lines (appearing almost superimposed on the solid ones) are similar neuron's distribution rather than from the data itself; vertical lines in $\boldsymbol{A}$ and $\boldsymbol{B}$ indicate the theoretical means of these distributions. $400 \mathrm{~ms}$ before to $500 \mathrm{~ms}$ after distractor onset for the target in RF condition $(\boldsymbol{A}, \boldsymbol{C})$, and from $470 \mathrm{~ms}$ before to $30 \mathrm{~ms}$ after distractor onset for the distractor in RF condition $(B, D)$.

increase in mean (with near-constant variance on average) as a reduction in variance below that expected on the basis of the Poisson model. In contrast, for the distractor in RF condition, the reduction in Fano factor with increased expected reward (median decrease $=0.56 ; p=0.0078$, signed-rank test; $n=46$ ) appears to mainly be a result of a marginally statistically insignificant reduction in variance (median decrease $=2.94 ; p=0.0795$, signedrank test; $n=46$ ) rather than a change in the mean spike count (median decrease $=0.25 ; p=0.8313$, signed-rank test; $n=46$ ) .

\section{Relationship to burstiness and regularity}

The negative-binomial model may emerge either from acrosstrial variations in the mean firing rate of a Poisson process or from variations in the properties of bursts in the spike train. We used the spike trains from the epochs used for the model fitting above to examine whether insight could be gained into the influence of burstiness and the regularity of interspike intervals on the Fano factor patterns we report here. The BRI (Compte et al., 2003; Anderson et al., 2011; see Materials and Methods) did not differ significantly across the four conditions (target in RF and distractor in RF with small and large reward) and had a mean value of $0.22(\mathrm{SEM}=0.02)$. This value is clearly significantly $>0$, but well below the thresholds for burstiness ( 1 and 2 ) used in the previous studies, indicating a consistent but only slight level of burstiness in LIP responses under these conditions. The BRI is correlated with the Fano factor after controlling for firing rate, 
both with the distractor in RF (partial correlations: small reward, $r=0.51, p=0.0004$; large reward, $r=0.64, p<0.0001)$ and with the target in $\mathrm{RF}$ (small reward, $r=0.48, p=0.0029$; large reward, $r=0.36, p=0.0306)$. Further, the change in Fano factor with expected reward is correlated with the accompanying change in BRI with the target in RF (partial correlation after factoring out the change in firing rate, $r=0.45, p=0.005$ ) and marginally so with the distractor in RF (partial correlation, $r=0.27, p=$ $0.0767)$. The data suggest that there may be a small level of burstiness in LIP responses (consistent with Maimon and Assad, 2009), and this burstiness can be correlated with the Fano factor.

We also examined the irregularity in the interspike intervals within the same epoch using two measures of the coefficient of variation that try to account for within-trial and across-trial variations in firing rate: CV2 (Holt et al., 1996); and CV (Softky and Koch, 1993). The mean values for these two measures were close to 1 , which is the expected value for a Poisson process, and there were only small differences between them across the four conditions $(\mathrm{CV}$ : mean $\pm \mathrm{SEM}=0.99 \pm 0.02 ; \mathrm{CV} 2$ : mean $\pm \mathrm{SEM}=$ $1.02 \pm 0.01$ ). A similar value is obtained (mean $\pm \mathrm{SEM}=1.03 \pm$ 0.02 ) using a modified version of $\mathrm{CV}$ that only analyzed firing rates between 10 and $40 \mathrm{~Hz}$, consistent with a previous report of LIP responses using this measure (Maimon and Assad, 2009). Fitting a gamma distribution to the rate-parsed interspike intervals (Softky and Koch, 1993; Maimon and Assad, 2009) indicated that spike trains were regular, with a median value of the shape parameter $(2.05)$ that is significantly $>1(p<0.0001)$ and is comparable to that in the previous report on LIP (1.95). This is important because one potential explanation for the linearly increasing relationship between the mean and the Fano factor in the target-mapping data relies on the presence of irregularity in the spike train, with values for the shape parameter $<1$ (Nawrot et al., 2008; see Discussion). We did not find any significant relationships between CV2 and the Fano factor, or in the changes in these measures with expected reward, even after controlling for the effects of mean firing rate (all $p>0.05$ ).

\section{Assessing the fit of the general linear model using the negative-binomial model}

The negative-binomial model not only fits the spike count distributions in the cued reward dataset but can also capture the linear relationship between the mean spike count and the Fano factor seen in the target-mapping data (Lindén and Mäntyniemi, 2011; see Discussion). Because of the small number of trials for each target location in the target-mapping task, we did not attempt to estimate the best-fitting parameters of the negative-binomial model for this dataset because such estimates based on small samples can be severely affected by estimation bias, and well developed methods to overcome this bias do not exist at the moment. We, however, used the model to get an idea of how well the multiplicative general linear model fit the observed data. While the general linear model appears to extract the correct slopes and intercepts on average and capture the trends in the population (Fig. 4), it only explained a relatively small proportion of the variance for each neuron (mean $\pm \mathrm{SEM} r^{2}=0.18 \pm 0.02$ ). However, this must be evaluated in the light of the relatively few number of trials at each target-RF distance and the variability inherent in neuronal spiking, which would both limit the $r^{2}$ values even if the data truly emerged from the model and the only variability in the data comes from the stochasticity inherent in the model. To account for these factors, we calculated the $r^{2}$ values that one could expect if the negative-binomial model really held true. We estimated the parameters of the best-fitting negative-binomial distribution for each target location from the slope and intercept value for that target-RF distance indicated by the general linear model (see Materials and Methods). Across all neurons and target-RF distances $\left(>5^{\circ}\right), 77.2 \%$ had a variance greater than or equal to the mean. We performed the estimation for 68 of the 72 neurons that showed a variance greater than or equal to the mean for at least half the target locations, allowing valid negativebinomial parameters to be estimated (even if they were biased). We then simulated the same number of trials as in the data for each target location using the parameters estimated above and repeated this process 500 times to calculate a distribution for the $r^{2}$ value that would emerge if the data were really generated from the proposed model. We could then estimate the (one-tailed) $p$ value that an $r^{2}$ value as small as that in the actual data could emerge from the simulated model; this value is clearly nonsignificant (mean $\pm \operatorname{SEM} p=0.62 \pm 0.03 ; n=68$ neurons). In none of the neurons is $p<0.025$ (indicating that the model $r^{2}$ value was significantly less than the data $r^{2}$ value), and in only three of the neurons is $p>0.975$ (indicating that the model $r^{2}$ is significantly greater than the data $r^{2}$ ). This proportion is consistent with chance and strongly suggests that the apparently low $r^{2}$ values are due to the small number of trials and not to the inefficacy of the general linear model.

\section{Behavioral significance of the Fano factor for saccades}

Prior reports from other brain areas have indicated that the Fano factor in the dorsal premotor cortex, FEF, and V4 (Churchland et al., 2006; Steinmetz and Moore, 2010; Chang et al., 2012) is associated with changes in behavioral response time. Such an effect is also visible in our cued reward data. We showed previously that increases in expected reward are associated with significantly shorter latency saccades on correct trials as well as a decreased proportion of saccades toward the distractor, and these behavioral effects are accompanied by changes in mean spike rate (Falkner et al., 2010) as well as the reduction in Fano factor that we have shown above. However, within each reward condition (large or small expected reward), we did not find a significant difference in the Fano factors for trials with saccades with latencies shorter or longer than the median. We reasoned that such an effect might be more detectable in the data from a memoryguided saccade task (Fig. $7 A, B$ ) where saccadic latencies are typically longer and more variable compared with the visually guided saccade task. We examined the Fano factor (Fig. 7C,E) and mean spike rate (Fig. $7 D$ ) when the monkey made a memoryguided saccade to a single remembered location in the suppressive surround. The task timing was identical to that in the cued reward task, and a flashed distractor was presented at the RF center. We found that the Fano factor was larger for trials with saccades with longer latencies (greater than the median) toward the surround (population PSTF: Fig. 7E; median increase during the $200 \mathrm{~ms}$ period before the disappearance of the fixation point (the go-cue) $=0.32 ; p=0.0247$, signed-rank test; $n=39$ ). However, the mean spike rate during this epoch was not significantly different between these two sets of trials (population PSTH: Fig. $7 D$; median increase $=0.60 ; p=$ 0.1391 , signed-rank test; $n=39$ ).

\section{Discussion}

We show that the Fano factor in LIP is maximally reduced for neurons representing the saccade goal. The reduction in Fano factor becomes smaller as the saccade target moves away from the $\mathrm{RF}$. This creates a variability valley in the LIP priority map at the location of the saccade goal in addition to the well known peak in 
A

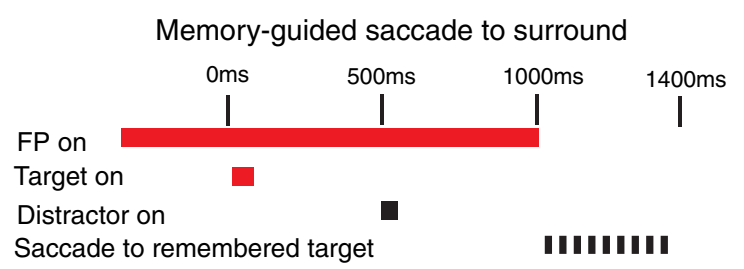

D

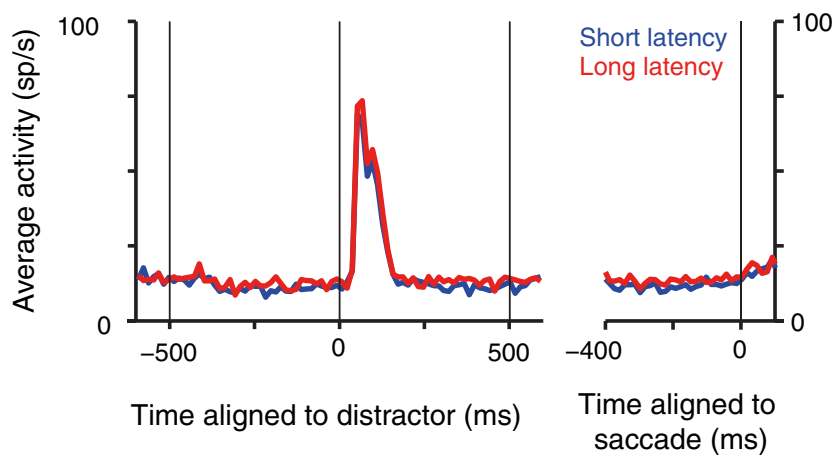

B

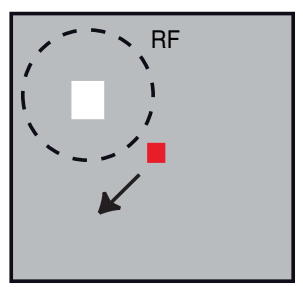

C

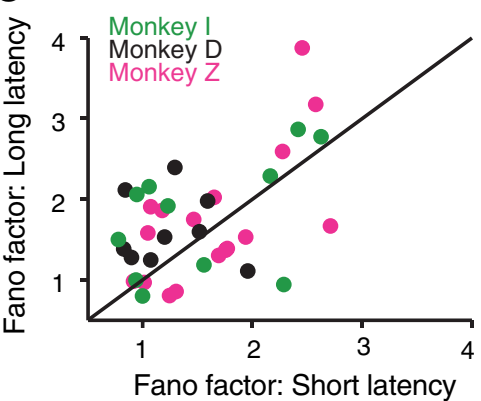

E
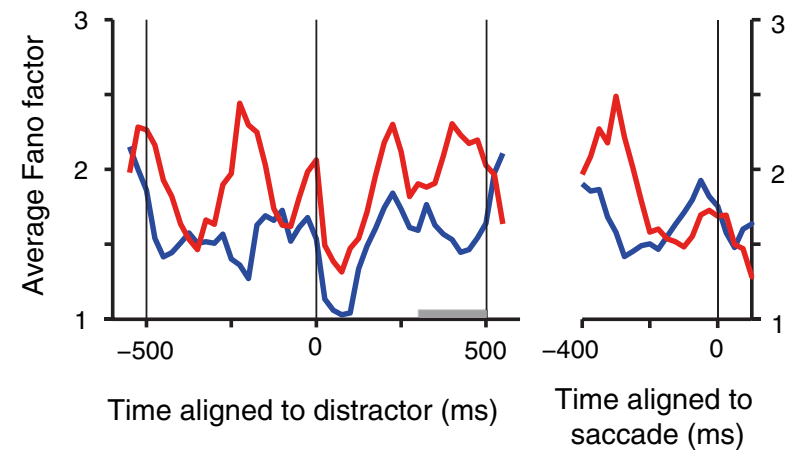

Figure 7. Larger neuronal Fano factor is associated with longer-latency memory-guided saccades to the surround. The PSTFs and PSTHs show data for saccades with latencies longer than the median in red, and for saccades with latencies shorter than the median in blue. Graph format as in Figure 5. $\boldsymbol{A}, \boldsymbol{B}$, Timing $(\boldsymbol{A})$ and schematic diagram of task (B). $\boldsymbol{C}-\boldsymbol{E}$, Population PSTHs for the mean spike rate $(\boldsymbol{D})$ and PSTFs for the Fano factor $(\boldsymbol{E})$, as well as the scatter plot for Fano factor associated with short-latency (abscissa) and long-latency saccades (ordinate) in $\boldsymbol{C}$ (evaluated $200 \mathrm{~ms}$ before the go-cue; gray window in $\boldsymbol{E}$ ). The data in $\boldsymbol{C}$ and $\boldsymbol{E}$ indicate a larger Fano factor for long-latency saccades; this is statistically significant (signed-rank test, $p=0.0247 ; n=39)$. Two points in $\boldsymbol{C}(x$-axis, $1.60,8.27 ; y$-axis, 2.02, 6.34) were omitted from the display for better visibility.

mean spike rate. The broad extent over which the variability decrease is observed reconfirms the broad-ranging nature of the LIP surround. The Fano factor increases more sharply with mean spike count for neurons with RF centers farther away from the saccade target, consistent with a multiplicative interaction between target-RF distance and spike count. The Fano factor is behaviorally significant: increasing expected reward leads to both a reduced Fano factor as well as shorter saccade latencies and fewer distractor-directed erroneous saccades; and memoryguided saccade trials culminating in longer-latency saccades are associated with larger Fano factors in neurons representing the surround.

Our results are consistent with the hypothesis that changes in Fano factor reflect both stimulus and behavioral engagement in LIP. This hypothesis is also suggested by recent findings showing that sensory stimulation in many cortical areas (Churchland et al., 2010; Oram, 2011), as well as attention in V4 (Mitchell et al., 2007; Cohen and Maunsell, 2009) and task engagement in the prefrontal cortex (Hussar and Pasternak, 2010), reduce the Fano factor. Neurons whose RFs overlap the target and have the lowest Fano factors are defined as being the most engaged by the stimulus. Similarly, increases in motivation or arousal correspond to increased behavioral engagement in the cued reward task and memory-guided saccade to surround task, as evidenced by shorter saccade latencies, and are accompanied by reductions in Fano factor. Moreover, while both stimulus and behavioral engagement can have a variety of effects on mean spike rate (increase, decrease, or no change), they appear to consistently reduce the Fano factor.

Our data do not indicate a specific biophysical basis for the Fano factor reductions. We speculate, extending the work of
Churchland et al. (2010), that engagement may reflect the strength of inputs received by the neuron in the task, such that the excitatory and inhibitory inputs received by each neuron counteract each other when determining the mean spike count but act in concordance to reduce the Fano factor. Farther targets reduce the Fano factor less because they evoke less input to the recorded LIP neurons. Visual input (as in the target-mapping task), and modulatory top-down input (as in the cued reward and memoryguided saccade tasks) may both act via this mechanism to reduce the Fano factor from the level set by ongoing activity in the brain. The reduction in variability may result from the interaction between external stimulus drive and internal dynamics in neuronal networks (Rajan et al., 2010; Litwin-Kumar and Doiron, 2012) or changes in input spike synchrony (Sripati and Johnson, 2006). However, recent reports indicate that recurrent excitatory inputs that may be responsible for maintained delay-period activity or attentional allocation in FEF may not lead to Fano factor reductions (Chang et al., 2012; Purcell et al., 2012).

\section{Relationship of Fano factor to mean spike count:}

\section{interpretation based on the negative-binomial model}

Our data show that Fano factors are well above 1 and increase linearly with the mean spike count at a given target-RF distance. Both these effects represent deviations from the basic Poisson model, where the Fano factor is always 1. Both these deviations can be captured by a negative-binomial model, which is an extension of the Poisson model that is commonly used to model overdispersion. This extension may be justified in the following two ways (Boswell and Patil, 1970). (1) If the mean spike count on each trial for a Poisson process is sampled randomly from a gamma distribution with parameters $k$ and $\theta$, then the resultant 
distribution of spike counts across trials will be a negativebinomial with the same $k$ and $\theta$ parameters. This approach based on trial-by-trial variations in firing rate has been used before to characterize Fano factors $>1$ in hippocampal neurons (Olypher et al., 2002) and recently in LIP itself to differentiate between different models of decision making (Shadlen and Newsome, 1998; Churchland et al., 2011). Specifically, this interpretation is conceptually similar to that in Churchland et al. (2011), but is more specific in that it requires a Poisson counting process with a gamma-distributed mean spike count on each trial and can, therefore, predict the full distribution of spike counts. In contrast, the approach of Churchland et al. (2011) restricts itself to estimating the variance of the mixing distribution up to an unknown offset value that depends on spike count, assuming only a quasi-Poisson counting process (with an unknown proportionality constant) whose mean rate varies from trial to trial. (2) A Poisson process where each event is a burst rather than a spike, and the number of spikes within a burst is distributed according to a logarithmic distribution leads to a negative-binomial distribution of spike counts. We find evidence for a small degree of burstiness in LIP, consistent with the work of Maimon and Assad (2009), and interactions between burstiness and Fano factor have been reported before from the forebrain nucleus of a songbird (Kao et al., 2008). However, differentiating between these two generating models is nontrivial, and is beyond the scope of our dataset and analyses.

Using the negative-binomial model, the linear relationship that we observe between $F$ and $M$ in the target-mapping task at a fixed target-RF distance $(F=a M+b$; Fig. 4$)$ can be captured very naturally. When $\theta$ varies while $k$ remains constant, and thus all the variation in mean spike count comes from the variation in $\theta$, the measured Fano factor varies linearly with mean spike count with a slope of $1 / k$ and an intercept of 1 ; a similar situation is obtained even when $k$ and $\theta$ remain constant and the mean spike count and Fano factor vary due to sampling variation. More generally, if the shape parameter $k$ and the scale parameter $\theta$ vary in a correlated manner such that $\theta=a M+b-1=F_{\text {regression }}-1$ and $k=M / \theta$ (Lindén and Mäntyniemi, 2011), then the Fano factor varies linearly with the mean spike count with a possibly nonzero intercept. Using this model, within each target-RF distance zone (Fig. 4), the scale parameter $\theta$ therefore behaves similarly to the Fano factor and increases steadily with target-RF distance, while $k$ decreases with target-RF distance if the mean spike count remains constant. In the cued reward task, when the monkey expects a larger reward, $\theta$ decreases and $k$ increases both when the target is in the RF and when the distractor is in the RF (since the Fano factor goes down in both cases but the spike rate either goes up or stays unchanged). In the two interpretations discussed above, these variations in the mean and Fano factor with target-RF distance and motivation then correspond to either variations in the shape and scale of the gamma function or to variations in the rate of burst occurrence and the distribution of the number of spikes within a burst.

In principle, an alternative explanation for the linear relationship between the Fano factor and mean spike count could emerge from a combination of the short (up to $500 \mathrm{~ms}$ long) measurement windows combined with a spike train process that is substantially more irregular than a Poisson process (Nawrot et al., 2008). However, our own measurements, as well as prior reports of regular "pulse-like" firing in LIP neurons (Maimon and Assad, 2009), suggest that LIP spike trains may, if anything, be more regular than a Poisson process, and so the necessary assumptions for this explanation may not hold. The explanation based on the negative-binomial model appears to be more satisfactory because this model provides a good fit to the data from the cued reward task. In addition, other deviations from a Poisson process (like interspike interval correlations) may also have currently uncharacterized effects on spike count variability. However, the effects we observe are clearly not the result of refractory-period-related changes, since such changes would lead to reductions of Fano factor with increasing spike rate.

Our results provide a starting framework to understand the previously observed unsystematic relationships between spike rate and Fano factor in different brain areas: prior analyses have primarily restricted themselves to attempting to control for the influence of spike rate by comparing Fano factors for neurons with matched spike rates (Churchland et al., 2010). Our results add to the literature indicating that the Fano factor reveals information additional to that in the mean spike rate (Churchland et al., 2010; Hussar and Pasternak, 2010; Steinmetz and Moore, 2010). Changes in across-trial variability have been reported to correlate with aging (Garrett et al., 2010) and with the presence of autism (Dinstein et al., 2012) in human fMRI recordings.

\section{Implications for LIP priority map}

We find an extended spatial gradient of Fano factor surrounding the saccade target during a visually guided saccade task. The broad-ranging LIP spatial surround can then not only sharpen the priority map by suppressing mean spike rates, but can also improve priority map precision by reducing the Fano factor (Paradiso, 1988). Even the reduced variability in response to distractor onset could contribute to improve filtering of the distractor. The association in our data between saccade latency to the surround and the Fano factor suggests that the variability of responses in neurons whose RFs surround the target may have functional consequences for behavior (Steinmetz and Moore, 2010). Our findings provide a clear target for network models of priority map representations.

\section{References}

Anderson EB, Mitchell JF, Reynolds JH (2011) Attentional modulation of firing rate varies with burstiness across putative pyramidal neurons in macaque visual area V4. J Neurosci 31:10983-10992. CrossRef Medline

Averbeck BB, Lee D (2003) Neural noise and movement-related codes in the macaque supplementary motor area. J Neurosci 23:7630-7641. Medline

Bisley JW (2011) The neural basis of visual attention. J Physiol 589:49-57. CrossRef Medline

Bisley JW, Goldberg ME (2010) Attention, intention, and priority in the parietal lobe. Annu Rev Neurosci 33:1-21. CrossRef Medline

Boswell MT, Patil GP (1970) Chance mechanisms generating the negative binomial distributions. In: Random counts in models and structures ( $\mathrm{Pa}-$ til GP, ed), pp 1-22. University Park, PA: Pennsylvania State UP.

Chang MH, Armstrong KM, Moore T (2012) Dissociation of response variability from firing rate effects in frontal eye field neurons during visual stimulation, working memory, and attention. J Neurosci 32:2204-2216. CrossRef Medline

Churchland AK, Kiani R, Chaudhuri R, Wang XJ, Pouget A, Shadlen MN (2011) Variance as a signature of neural computations during decision making. Neuron 69:818-831. CrossRef Medline

Churchland MM, Yu BM, Ryu SI, Santhanam G, Shenoy KV (2006) Neural variability in premotor cortex provides a signature of motor preparation. J Neurosci 26:3697-3712. CrossRef Medline

Churchland MM, Yu BM, Cunningham JP, Sugrue LP, Cohen MR, Corrado GS, Newsome WT, Clark AM, Hosseini P, Scott BB, Bradley DC, Smith MA, Kohn A, Movshon JA, Armstrong KM, Moore T, Chang SW, Snyder LH, Lisberger SG, Priebe NJ, et al (2010) Stimulus onset quenches neural variability: a widespread cortical phenomenon. Nat Neurosci 13:369378. CrossRef Medline

Cohen MR, Maunsell JH (2009) Attention improves performance primarily 
by reducing interneuronal correlations. Nat Neurosci 12:1594-1600. CrossRef Medline

Compte A, Constantinidis C, Tegner J, Raghavachari S, Chafee MV, Goldman-Rakic PS, Wang XJ (2003) Temporally irregular mnemonic persistent activity in prefrontal neurons of monkeys during a delayed response task. J Neurophysiol 90:3441-3454. CrossRef Medline

Dinstein I, Heeger DJ, Lorenzi L, Minshew NJ, Minshew NJ, Malach R, Behrmann M (2012) Unreliable evoked responses in autism. Neuron 75:981-991. CrossRef Medline

Falkner AL, Krishna BS, Goldberg ME (2010) Surround suppression sharpens the priority map in the lateral intraparietal area. J Neurosci 30:1278712797. CrossRef Medline

Fecteau JH, Munoz DP (2006) Salience, relevance, and firing: a priority map for target selection. Trends Cogn Sci 10:382-390. CrossRef Medline

Garrett DD, Kovacevic N, McIntosh AR, Grady CL (2010) Blood oxygen level-dependent signal variability is more than just noise. J Neurosci 30: 4914-4921. CrossRef Medline

Holt GR, Softky WR, Koch C, Douglas RJ (1996) Comparison of discharge variability in vitro and in vivo in cat visual cortex neurons. J Neurophysiol 75:1806-1814. Medline

Hussar C, Pasternak T (2010) Trial-to-trial variability of the prefrontal neurons reveals the nature of their engagement in a motion discrimination task. Proc Natl Acad Sci U S A 107:21842-21847. CrossRef Medline

Kao MH, Wright BD, Doupe AJ (2008) Neurons in a forebrain nucleus required for vocal plasticity rapidly switch between precise firing and variable bursting depending on social context. J Neurosci 28:1323213247. CrossRef Medline

Lindén A, Mäntyniemi S (2011) Using the negative binomial distribution to model overdispersion in ecological count data. Ecology 92:1414-1421. CrossRef Medline

Litwin-Kumar A, Doiron B (2012) Slow dynamics and high variability in balanced cortical networks with clustered connections. Nat Neurosci 15: 1498-1505. CrossRef Medline

Louie K, Grattan LE, Glimcher PW (2011) Reward value-based gain control: divisive normalization in parietal cortex. J Neurosci 31:10627-10639. CrossRef Medline

Maimon G, Assad JA (2009) Beyond Poisson: increased spike time regularity across primate parietal cortex. Neuron 62:426 -440. CrossRef Medline

Marcos E, Pani P, Brunamonti E, Deco G, Ferraina S, Verschure P (2013) Neural variability in premotor cortex is modulated by trial history and predicts behavioral performance. Neuron 78:249-255. CrossRef Medline

McAdams CJ, Maunsell JH (1999) Effects of attention on the reliability of individual neurons in monkey visual cortex. Neuron 23:765-773. CrossRef Medline

Mitchell JF, Sundberg KA, Reynolds JH (2007) Differential attentiondependent response modulation across cell classes in macaque visual area V4. Neuron 55:131-141. CrossRef Medline

Nawrot MP, Boucsein C, Rodriguez Molina V, Riehle A, Aertsen A, Rotter S (2008) Measurement of variability dynamics in cortical spike trains. J Neurosci Methods 169:374-390. CrossRef Medline

Olypher AV, Lánský P, Fenton AA (2002) Properties of the extra-positional signal in hippocampal place cell discharge derived from the overdispersion in location-specific firing. Neuroscience 111:553-566. CrossRef Medline

Oram MW (2011) Visual stimulation decorrelates neuronal activity. J Neurophysiol 105:942-957. CrossRef Medline

Oram MW, Hatsopoulos NG, Richmond BJ, Donoghue JP (2001) Excess synchrony in motor cortical neurons provides redundant direction information with that from coarse temporal measures. J Neurophysiol 86: 1700-1716. Medline

Orer HS, Das M, Barman SM, Gebber GL (2003) Fractal activity generated independently by medullary sympathetic premotor and preganglionic sympathetic neurons. J Neurophysiol 90:47-54. CrossRef Medline

Osborne LC, Bialek W, Lisberger SG (2004) Time course of information about motion direction in visual area MT of macaque monkeys. J Neurosci 24:3210-3222. CrossRef Medline

Paradiso MA (1988) A theory for the use of visual orientation information which exploits the columnar structure of striate cortex. Biol Cybern 58: 35-49. CrossRef Medline

Purcell BA, Heitz RP, Cohen JY, Schall JD (2012) Response variability of frontal eye field neurons modulates with sensory input and saccade preparation but not visual search salience. J Neurophysiol 108:2737-2750. CrossRef Medline

Rajan K, Abbott LF, Sompolinsky H (2010) Stimulus-dependent suppression of chaos in recurrent neural networks. Physical Rev E Stat Nonlin Soft Matter Phys 82:011903. CrossRef

Rao PSRS, Rao JNK (1971) Small sample results for ratio estimators. Biometrika 58:625-630. CrossRef

Rao TJ (2002) Mean of ratios or ratio of means or both? J Stat Plan Infer 102:129-138. CrossRef

Scobey RP, Gabor AJ (1989) Orientation discrimination sensitivity of single units in cat primary visual cortex. Exp Brain Res 77:398-406. CrossRef Medline

Shadlen MN, Newsome WT (1998) The variable discharge of cortical neurons: implications for connectivity, computation, and information coding. J Neurosci 18:3870-3896. Medline

Softky WR, Koch C (1993) The highly irregular firing of cortical cells is inconsistent with temporal integration of random EPSPs. J Neurosci 13: 334-350. Medline

Sripati AP, Johnson KO (2006) Dynamic gain changes during attentional modulation. Neural Comput 18:1847-1867. CrossRef Medline

Steinmetz NA, Moore T (2010) Changes in the response rate and response variability of area V4 neurons during the preparation of saccadic eye movements. J Neurophysiol 103:1171-1178. Medline

Teich MC, Heneghan C, Lowen SB, Ozaki T, Kaplan E (1997) Fractal character of the neural spike train in the visual system of the cat. J Opt Soc Am A Opt Image Sci Vis 14:529-546. CrossRef Medline

Tolhurst DJ, Movshon JA, Thompson ID (1981) The dependence of response amplitude and variance of cat visual cortical neurones on stimulus contrast. Exp Brain Res 41:414-419. Medline

Vogels R (1990) Population coding of stimulus orientation by striate cortical cells. Biol Cybern 64:25-31. CrossRef Medline 